\title{
Daniele Faenzi
}

\section{Bundles over Fano threefolds of type $V_{22}$}

Received: 14 November 2003 / Revised: 19 October 2004 / Published online: 29 May 2006

(c) Springer-Verlag 2006

\begin{abstract}
An explicit resolution of the diagonal over $V_{22}$ is given, using some observations about instanton bundles with $c_{2}=3$. Different descriptions of $V_{22}$ are interpreted in terms of mutations of vector bundles.
\end{abstract}

Keywords Fano threefolds · Twisted cubics · Plane quartics · Sums of powers · Nets of quadrics - Instanton bundles - Exceptional collections · Mutations · Helices

Mathematics Subject Classification (2000) 14J60, 14D20, 14F05, 14J30, 14J45

\section{Introduction}

Smooth complex Fano threefold with Picard number 1 and with no harmonic 1, 2 forms play a special rôle. According to Iskosvskih's classification $[15,16]$, there exist four classes of such varieties, namely, $\mathbb{P}^{3}, Q_{3}, V_{5}$ and $V_{22}$, respectively, of index 4, 3, 2 and 1 , where the genus-12 variety $V_{22}$ is the only one with non-trivial infinitesimal deformations. Their degree, respectively $1,2,5$ and 22, is maximal in each index class; they are all rational and deformation equivalent to a smooth orbit closure of the group $\mathrm{SL}(2, \mathbb{C})$. Moreover, their $K$-theory group is isomorphic to $\mathbb{Z}^{4}$. The geometry of these varieties has been studied in a great number of papers, and we refer to [17] for their exhaustive treatment and to [2, 22, 27] for more important results.

Here we will study the variety $V_{22}$ in terms of vector bundles it and we will prove the following result.

D. Faenzi (ख)

Dipartimento di Matematica “U. Dini”, Università di Firenze, Viale Morgagni 67/a, I-50134, Florence, Italy

E-mail: faenzi@math.unifi.it, Url: http://www.math.unifi.it/ faenzi/ 
Theorem 1.1 The general variety $X$ of type $V_{22}$ admits the resolution of the diagonal

$$
0 \rightarrow G_{3} \otimes G^{3} \rightarrow G_{2} \otimes G^{2} \rightarrow G_{1} \otimes G^{1} \rightarrow G_{0} \otimes G^{0} \rightarrow \mathcal{O}_{\Delta} \rightarrow 0
$$

where $\left(G_{3}, \ldots, G_{0}\right)$ (respectively $\left.\left(G^{3}, \ldots, G^{0}\right)\right)$ is an exceptional collection of stable aCM bundles of rank 2, 3, 4, 1 (respectively of rank 2, 5, 3, 1).

This gives an analogue of Berlinson's theorem over the projective space, see [3]. Further instances of this fact were found, e.g. by Kapranov in [18], by Canonaco for weighted projective spaces in [6], by Orlov in [26] for the threefold $V_{5}$, by the author in [11] still for the threefold $V_{5}$.

The main tools are the results obtained by Schreyer in [27] and by Mukai in [21] involving nets of quadrics, 3-instanton bundles on $\mathbb{P}^{3}$ and nets of alternating 2 -forms. It will turn out that mutations of the bundles $G_{i}$ of the above theorem are closely related to the different descriptions of the threefold $V_{22}$.

The paper is organized as follows. In Sect. 2, we give the basic definitions and lemmas. In Sect. 3, we provide the first description of $V_{22}$ by means of nets of quadrics, recall its relation with the moduli space of twisted cubics and with 3 -instanton bundles on $\mathbb{P}^{3}$. Section 4 takes care of the definition of $V_{22}$ via nets of alternating 2-forms and contains the technical core of the paper i.e. Theorem 4.5. This theorem is crucial in Proposition 6.4, which in turn is the key to prove Theorem 7.2, our main result. Section 5 is devoted to the description of $V_{22}$ via polar hexagons to a plane quartic. In Sect. 6 we give several results concerning bundles on $X$ and describe the homomorphism groups between them, while in Sect. 7 we state precisely and prove the main result (cfr. Theorem 7.2), together with some corollaries (cfr. Corollary 7.3 and 7.4). Finally in Sect. 8 we draw some remarks, including helices and the Mukai-Umemura case, i.e. a threefold of type $V_{22}$ with an SL(2) quasi-homogeneous structure.

Remark After this paper was finished, the author learned of the existence of an interesting preprint by Alexander Kuznetsov [20], where similar questions are investigated, although making use of different methods.

\section{Generalities}

We will always assume that the ambient variety $X$ is a compact complex algebraic smooth variety with $\operatorname{Pic}(X) \simeq \mathbb{Z}=\left\langle\mathcal{O}_{X}(1)\right\rangle, \mathcal{O}_{X}(1)$ being a very ample line bundle.

Definition 2.1 For a pair of vector bundles $\mathcal{F}$ and $\mathcal{G}$ on a variety $X$, define $p_{\mathcal{F}, \mathcal{G}}: \operatorname{Hom}(\mathcal{F}, \mathcal{G}) \otimes \mathcal{F} \rightarrow \mathcal{G}$ and $i_{\mathcal{F}, \mathcal{G}}: \mathcal{F} \rightarrow \operatorname{Hom}(\mathcal{F}, \mathcal{G})^{*} \otimes \mathcal{G}$ as the canonical evaluations. If $p_{\mathcal{F}, \mathcal{G}}$ (respectively $\left.i_{\mathcal{F}, \mathcal{G}}\right)$ ) is surjective (respectively injective) define the left mutation $\mathrm{L}_{\mathcal{F}} \mathcal{G}=\operatorname{ker}\left(p_{\mathcal{F}, \mathcal{G}}\right)$ (respectively the right mutation $\left.\mathrm{R}_{\mathcal{G}} \mathcal{F}=\left(\operatorname{coker} i_{\mathcal{F}, \mathcal{G}}\right)\right)$.

We refer to the book [1] useful properties of mutations, to [7] and [12] for their original use over projective spaces.

For any complex vector space $V$ denote by $1_{V}$ (respectively by $\chi_{V}$ ) the identity map of $V$ (respectively the canonical map $V^{*} \otimes V \rightarrow \mathbb{C}$ ). We write $S^{i}$, where $i$ 
is a finite sequence of nondecreasing integers, for the Schur functor associated to the Young tableau defined by the partition given by $i$. More precisely, the tableau defined by $i$ has $i_{j}$ boxes on the $j$-th row. For example $\mathrm{S}^{j} V$, where $j$ is an integer and $V$ is a vector space, is the $j$-th symmetric power of $V$.

Definition 2.2 Given a sheaf $\mathcal{F}$ over $X$, we say that $\mathcal{F}$ is aCM (for arithmetically Cohen-Macaulay) if $\mathrm{H}^{p}(X, \mathcal{F}(t))=0$, for all $t \in \mathbb{Z}$ and for $0<p<\operatorname{dim}(X)$.

We will write $\mathrm{H}^{p}(-)$ or $\operatorname{Hom}(-,-)$ instead of $\mathrm{H}^{p}(X,-)$ or $\operatorname{Hom}_{X}(-,-)$ unless the ambient variety $X$ is not clear from the context.

Given a subvariety $Z \subset X$ we denote its ideal sheaf by $J_{Z, X}$ and, by abuse of notation, the ideal of $Z$ in the coordinate ring of $X$.

Definition 2.3 A variety $X$ of type $V_{22}$ is a smooth projective threefold with $\operatorname{Pic}(X)=\left\langle\mathcal{O}_{X}(1)\right\rangle=\left\langle\omega_{X}^{*}\right\rangle$ and $\operatorname{deg}\left(\mathcal{O}_{X}(1)\right)=22$.

We refer to [14] for the definition of stability of bundles, in the sense of Mumford-Takemoto, with respect to the positive generator $c_{1}\left(\mathcal{O}_{X}(1)\right)$ of $\operatorname{Pic}(X)$.

Recall from [17] that the Chow ring $\mathrm{CH}(X)$ is isomorphic to $\mathbb{Z}^{4}$, where $\mathrm{CH}^{2}(X)$ (respectively $\mathrm{CH}^{3}(X)$ ) is generated by the class of a line (respectively of a point) in $X$.

Given a vector bundle $\mathcal{F}$ on $X$ we denote its Chern classes by $c_{i}(\mathcal{F})$, for $1 \leq$ $i \leq 3$ by $c_{i} \in \mathbb{Z}$, meaning $c_{i}(\mathcal{F})=c_{i} \xi_{i}$, where $\xi_{1}=c_{1}\left(\mathcal{O}_{X}(1)\right), \xi_{2}$ is the cohomology class of a line in $X$ and $\xi_{3}$ is the cohomology class of a point in $X$. Denote by $\mu(\mathcal{F})$ the rational number $c_{1}(\mathcal{F}) / \operatorname{rk}(\mathcal{F})$, called the slope of $\mathcal{F}$. We say that a bundle $\mathcal{F}$ is normalized if $-1<\mu(\mathcal{F}) \leq 0$. We write $\mathcal{F}_{n}$ for the unique normalized twist of $\mathcal{F}$. The proof of following lemma can be adapted from $\mathbb{P}^{n}$ since $\operatorname{Pic}(X) \simeq \mathbb{Z}$, see [25].

Lemma 2.4 (Hoppe) Let $\mathcal{F}$ be a rank $r$ vector bundle on $X$. Then $\mathcal{F}$ is stable if $\mathrm{h}^{0}\left(\left(\wedge^{p} \mathcal{F}\right)_{n}\right)=0$ for $1 \leq p<r$.

\section{Nets of dual quadrics and 3-instanton bundles on $\mathbb{P}^{3}$}

Let $A \simeq \mathbb{C}^{4}$ and $B \simeq \mathbb{C}^{3}$ be complex vector spaces, and let $R=\mathbb{C}[A]=$ $\mathbb{C}\left[x_{0}, \ldots, x_{3}\right]$ and $T=\mathbb{C}[B]$ be polynomial algebras over them. Considering the dual ring $R^{*}=\mathbb{C}\left[A^{*}\right]$ we have $R^{*} \simeq \mathbb{C}\left[\partial_{0}, \ldots, \partial_{3}\right]$. Then define the apolarity action of $R^{*}$ on $R$ by differentiation $\partial^{i}\left(x_{j}\right)=i ! j / i x_{j-i}$, where $i, j$ are multiindices and $\partial^{i}\left(x_{j}\right)=0$ if $j \ngtr i$. Then for $\partial \in \mathrm{S}^{i} A^{*}$ we have the apolarity map $\partial: \mathrm{S}^{j} A \rightarrow \mathrm{S}^{j-i} A$. In the same way $T^{*}$ acts on $T$ by apolarity and we have perfect pairings between degree $d$ polynomials over $R$ (respectively over $T$ ) and degree $d$ differential operators over $R$ (respectively over $T$ ).

We define the variety $\mathrm{H}$ to be the irreducible component of $\operatorname{Hilb}_{3 t+1}(\mathbb{P}(A))$ containing rational normal cubics in $\mathbb{P}(A)$, as constructed in [10]. The open subset $\mathrm{H}_{c}$ consisting of points which are Cohen-Macaulay embeds in $\mathbb{G}\left(\mathbb{C}^{3}, \mathrm{~S}^{2} A\right)$ by means of the vector bundle $U_{\mathrm{H}}^{*}$ whose fiber over $[\Gamma] \in \mathrm{H}_{c}$ is $\operatorname{Tor}_{1}^{R}\left(R / J_{\Gamma, \mathbb{P}^{3}}, \mathbb{C}\right)_{2} \simeq$ $\mathbb{C}^{3}$. Equivalently, we associate to any $[\Gamma] \in \mathrm{H}_{c}$ the net of quadrics on $\mathbb{P}(A)$ vanishing on $\Gamma$. 
Moreover, there exists a rank-2 bundle on $\mathrm{H}_{c}$ whose fiber over $\Gamma$ is $\operatorname{Tor}_{2}^{R}\left(R / J_{\Gamma, \mathbb{P}^{3}}, \mathbb{C}\right)_{3} \simeq \mathbb{C}^{2}$. Namely we take the space of first-order syzygies of $J_{\Gamma, \mathbb{P}^{3}}$. We denote this bundle by $E_{\mathrm{H}}$.

Lemma 3.1 Over the variety $\mathrm{H}_{c}$, the bundle $U_{\mathrm{H}}^{*}$ (respectively the bundle $E_{\mathrm{H}}$ ) is globally generated with $\mathrm{H}^{0}\left(U_{\mathrm{H}}^{*}\right)^{*} \simeq \mathrm{S}^{2} A$ (respectively with $\mathrm{H}^{0}\left(E_{\mathrm{H}}^{*}\right)^{*} \simeq \mathrm{S}^{2,1} A=$ $\left.\operatorname{ker}\left(\mathrm{S}^{2} A \otimes A \rightarrow \mathrm{S}^{3} A\right)\right)$. The vector bundle $E_{\mathrm{H}}^{*}$ embeds $\mathrm{H}_{c}$ into $\mathbb{G}\left(\mathbb{C}^{2}, \mathrm{~S}^{2,1} A\right)=$ $\mathbb{G}\left(\mathbb{P}^{1}, \mathbb{P}^{19}\right)$.

We have the canonical isomorphisms $\operatorname{Hom}\left(E_{\mathrm{H}}, U_{\mathrm{H}}\right) \simeq A^{*}$, Hom $\left(\wedge^{2} U_{\mathrm{H}}, E_{\mathrm{H}}\right) \simeq A^{*}$ and $\mathrm{H}^{0}\left(\wedge^{2} U^{*}\right)^{*} \simeq \wedge^{2} \mathrm{~S}^{2} A \simeq \mathrm{S}^{3,1}$ A. The morphism $i_{\wedge^{2} U_{\mathrm{H}}, E_{\mathrm{H}}}$ is induced by the map $\wedge^{2} S^{2} A \rightarrow A \otimes S^{2,1} A$ in the diagram below

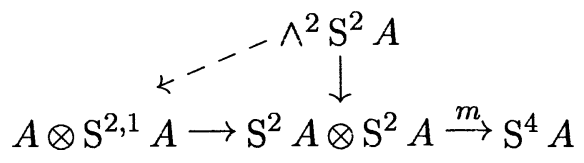

where the map $m$ is the multiplication in $\mathbb{C}[A]$ and the maps $\wedge^{2} S^{2} A \rightarrow$ $S^{2} A \otimes S^{2} A$ and $A \otimes S^{2,1} A \rightarrow S^{2} A \otimes S^{2} A$ are the canonical injections.

Proof Over a point [ $\Gamma]$ in $\mathrm{H}_{c}$ we take the minimal graded free resolution of $J_{\Gamma, \mathbb{P}^{3}}$ in degree 3 . This yields the exact sequence

$$
0 \leftarrow \mathrm{H}^{0}\left(J_{\Gamma, \mathbb{P}^{3}}(3)\right) \leftarrow A \otimes U_{\mathrm{H}, \Gamma} \leftarrow E_{\mathrm{H}, \Gamma} \leftarrow 0
$$

The above map $A \otimes U_{\mathrm{H}, \Gamma} \leftarrow E_{\mathrm{H}, \Gamma}$ is induced by $i_{E, U}$ and the isomorphism $\operatorname{Hom}\left(E_{\mathrm{H}}, U_{\mathrm{H}}\right) \simeq A^{*}$ is clear. Since $\operatorname{det}\left(U_{\mathrm{H}}\right) \simeq \mathcal{O}_{\mathrm{H}}(1)$ and $\operatorname{det}\left(E_{\mathrm{H}}\right) \simeq \mathcal{O}_{\mathrm{H}}(1)$ we have $\operatorname{Hom}\left(\wedge^{2} U_{\mathrm{H}}, E_{\mathrm{H}}\right) \simeq \operatorname{Hom}\left(E_{\mathrm{H}}^{*}, \wedge^{2} U_{\mathrm{H}}^{*}\right) \simeq \operatorname{Hom}\left(E_{\mathrm{H}}, U_{\mathrm{H}}\right) \simeq A^{*}$.

Since any quadratic form on $A$ contains a twisted cubic in $\mathrm{H}_{c}$, we have $\mathrm{H}^{0}\left(U_{\mathrm{H}}^{*}\right)$ $\simeq \mathrm{S}^{2} A^{*}$ and globalizing (2) we get $\mathrm{H}^{0}\left(E_{\mathrm{H}}^{*}\right)^{*} \simeq \mathrm{S}^{2,1} A=\operatorname{ker}\left(\mathrm{S}^{2} A \otimes A \rightarrow \mathrm{S}^{3} A\right)$. Since the $2 \times 2$ minors of the matrix $A \otimes U_{\mathrm{H}} \leftarrow E_{\mathrm{H}}$ in (2) define the twisted cubic $\Gamma, E_{\mathrm{H}}$ provides an embedding into $\mathbb{G}\left(\mathbb{C}^{2}, \mathrm{~S}^{2,1} A\right)$ and thus it is globally generated.

Finally, there are $\operatorname{SL}(A)$-equivariant isomorphisms $S^{2} A \otimes S^{2} A \simeq S^{2,2} A \oplus$ $S^{3,1} A \oplus S^{4} A, A \otimes S^{2,1} A \simeq S^{2,1,1} A \oplus S^{2,2} A \oplus S^{3,1} A, \wedge^{2} S^{2} A \simeq S^{2,1,1} A$. Then by Schur's Lemma the inclusion $\mathrm{S}^{2,1,1} A \hookrightarrow \mathrm{S}^{2} A \otimes \mathrm{S}^{2} A$ composes to zero with $m$ and therefore factors injectively through $A \otimes S^{2,1} A$, so the last statement is proved.

Definition 3.2 A net of dual quadrics $\Psi$ (parametrized by $B$ ) on $\mathbb{P}(A)$ is defined as a surjective map $\Psi: \mathrm{S}^{2} A \rightarrow B$. We also denote by $\Psi$ the composition $A \otimes A \rightarrow \mathrm{S}^{2} A \stackrel{\Psi}{\rightarrow}$ B. Let $\Psi^{\top}: B^{*} \rightarrow \mathrm{S}^{2} A^{*}$ be the transpose of $\Psi$ and let $V_{\Psi}=\operatorname{ker}(\Psi)$. For $[\Gamma] \in \mathrm{H}$ consider $J_{\Gamma, \mathbb{P} 3}$. Given a general net $\Psi$ define

$$
\begin{aligned}
X_{\Psi} & =\left\{[\Gamma] \in \mathrm{H} \subset \operatorname{Hilb}_{3 t+1}\left(\mathbb{P}^{3}\right) \mid \Psi\left(\mathrm{H}^{0}\left(J_{\Gamma, \mathbb{P}^{3}}(2)\right)\right)=0\right\} \\
& =\left\{[\Gamma] \in \mathrm{H} \subset \operatorname{Hilb}_{3 t+1}\left(\mathbb{P}^{3}\right) \mid \mathrm{H}^{0}\left(J_{\Gamma, \mathbb{P}^{3}}(2)\right) \subset V_{\Psi}\right\}
\end{aligned}
$$

the following result is due to Schreyer. 
Lemma 3.3 Given a general net of dual quadrics $\Psi: \mathrm{S}^{2} A \rightarrow B, X_{\Psi}$ is a Fano threefold of type $V_{22}$, equipped with a rank-2 vector bundle $E_{\mathrm{H}}$ and a rank-3 vector bundle $U_{\mathrm{H}}$.

Consider a net of dual quadrics $\Psi$ as defined in 3.2. We take the ideal $J^{\Psi}$ of polynomials in $R$ annihilated by $\Psi$, i.e.

$$
J^{\Psi}=\left\{p \in R \mid \Psi^{\top}(\beta)(p)=0, \forall \beta \in B^{*}\right\}
$$

where $\Psi^{\top}(\beta)$ sits in $\mathrm{S}^{2} A^{*}$ and for $\partial \in \mathrm{S}^{2} A^{*}, p \in \mathrm{S}^{2} A$ we define $\partial(p)$ by apolarity action as at the beginning of this section.

Definition 3.4 For general $\Psi$ define the Artinian ring $R^{\Psi}=R / J^{\Psi}$. Taking its minimal graded free resolution, put $V_{\Psi}^{i, j}=\operatorname{Tor}_{i}^{R}\left(R^{\Psi}, \mathbb{C}\right)_{j}$. As shown in [27, Lemma 4.1], the minimal graded free resolution of $R^{\Psi}$ reads

$$
\begin{aligned}
& 0 \leftarrow R / J^{\Psi} \leftarrow R \stackrel{p_{\Psi}}{\longleftarrow} V_{\Psi}^{1,2} \otimes R(-2) \stackrel{q_{\Psi}}{\longleftarrow} V_{\Psi}^{2,3} \otimes R(-3) \oplus V_{\Psi}^{2,4} \otimes R(-4) \stackrel{r_{\Psi}}{\longleftarrow} \\
& \stackrel{r_{\Psi}}{\longleftarrow} V_{\Psi}^{3,5} \otimes R(-5) \leftarrow\left(V_{\Psi}^{4,6}\right) \otimes R(-6) \leftarrow 0
\end{aligned}
$$

We have $R_{1}^{\Psi} \simeq A, R_{2}^{\Psi} \simeq B$ and $R_{d}^{\Psi}=0$ for $d \geq 3$.

Recall by [27, Corollary 4.3] that there is an isomorphism $\left(V_{\Psi}^{4,6}\right)^{*} \simeq V_{\Psi}^{2,4}$. The dimensions of the spaces $V_{\Psi}^{i, j}$ are the following

$$
\operatorname{dim}\left(V_{\Psi}^{1,2}\right)=7 \quad \operatorname{dim}\left(V_{\Psi}^{2,3}\right)=8 \quad \operatorname{dim}\left(V_{\Psi}^{2,4}\right)=3 \quad \operatorname{dim}\left(V_{\Psi}^{3,5}\right)=8
$$

There is a canonical isomorphism $V_{\Psi}^{1,2} \simeq V_{\Psi}=\operatorname{ker}(\Psi)$, indeed we have $V_{\Psi}^{1,2}=\left\{p \in \mathrm{S}^{2} A \mid \Psi^{\top}(\beta)(p)=0, \forall \beta \in B^{*}\right\} \simeq \operatorname{ker} \Psi$. Thus we will identify these spaces from now on.

Given a general net of dual quadrics $\Psi$ as in 3.2 and the ring $R^{\Psi}$ defined in 3.4 , consider the vector bundle $\operatorname{ker}\left(p_{\Psi}\right)$ over $\mathbb{P}(A)$ obtained sheafifying $p_{\Psi}$

$$
0 \rightarrow \operatorname{ker}\left(p_{\Psi}\right) \rightarrow V \otimes \mathcal{O}_{\mathbb{P}(A)}(-2) \stackrel{p_{\Psi}}{\longrightarrow} \mathcal{O}_{\mathbb{P}(A)} \rightarrow 0
$$

We get $\mathrm{H}^{0}\left(\operatorname{ker}\left(p_{\Psi}\right)(t)\right)=0$ for $t<3$.

Lemma 3.5 (Schreyer) Given a general net of dual quadrics $\Psi$ as in 3.2, the sheafification of the map $q_{\Psi}$ gives an instanton bundle $\mathcal{E}_{\Psi}$ defined by

$$
0 \rightarrow \mathcal{E}_{\Psi}(-5) \rightarrow V_{\Psi}^{2,3} \otimes \mathcal{O}_{\mathbb{P}(A)}(-3) \stackrel{q_{\Psi}}{\longrightarrow} \operatorname{ker}\left(p_{\Psi}\right) \rightarrow 0
$$

We have $c_{2}\left(\mathcal{E}_{\Psi}\right)=3$ and $\mathrm{h}^{1}\left(\mathcal{E}_{\Psi}(t)\right)=0$ except for $t=0,1,2$. Furthermore we have the canonical isomorphisms

$$
\begin{array}{lll}
\mathrm{H}^{1}\left(\operatorname{ker}\left(p_{\Psi}\right)\right) \simeq \mathbb{C} & \mathrm{H}^{1}\left(\operatorname{ker}\left(p_{\Psi}\right)(1)\right) \simeq A & \mathrm{H}^{1}\left(\operatorname{ker}\left(p_{\Psi}\right)(2)\right) \simeq B \\
\mathrm{H}^{1}\left(\mathcal{E}_{\Psi}(-1)\right) \simeq V_{\Psi}^{2,4} & \mathrm{H}^{1}\left(\mathcal{E}_{\Psi}\right) \simeq A^{*} & \mathrm{H}^{1}\left(\mathcal{E}_{\Psi}(1)\right) \simeq \mathbb{C}
\end{array}
$$


There exists an isomorphism $\mathrm{H}^{1}\left(\Omega_{\mathbb{P}(A)}^{1} \otimes \mathcal{E}_{\Psi}\right) \simeq V_{\Psi}^{3,5}$ and the vector space $V_{\Psi}^{3,5}$ is endowed with a canonical alternating duality. Finally, the instanton bundle $\mathcal{E}_{\Psi}$ is isomorphic to the cohomology of the monad

$$
\left(V_{\Psi}^{2,4}\right)^{*} \otimes \mathcal{O}_{\mathbb{P}(A)}(-1) \stackrel{r_{\Psi}}{\longrightarrow} V_{\Psi}^{3,5} \otimes \mathcal{O}_{\mathbb{P}(A)} \stackrel{r_{\Psi}^{\top}}{\rightarrow} V_{\Psi}^{2,4} \otimes \mathcal{O}_{\mathbb{P}(A)}(1)
$$

where the map $r_{\Psi}$ is defined by the minimal graded free resolution (4) and we $\operatorname{recall}\left(V_{\Psi}^{4,6}\right)^{*} \simeq V_{\Psi}^{2,4}$ and $\left(V_{\Psi}^{3,5}\right)^{*} \simeq V_{\Psi}^{3,5}$.

The relation between nets of quadrics and 3-instanton bundles has been thoroughly investigated by Gruson and Skiti in [13]. We give account of this in the following remark.

Remark 3.6 (Gruson-Skiti) For a general instanton bundle $\mathcal{E}$ on $\mathbb{P}(A)$ with $c_{2}(\mathcal{E})=3$, the homomorphism $\mathrm{H}^{1}(\mathcal{E}(-1)) \otimes A \rightarrow \mathrm{H}^{1}(\mathcal{E})$ gives a map $\Psi_{\mathcal{E}}^{\top}$ : $\mathrm{H}^{1}(E(-1)) \simeq \mathbb{C}^{3} \hookrightarrow A^{*} \otimes A^{*}$ since $\mathrm{H}^{1}(\mathcal{E}) \simeq A^{*}$. The map $\Psi_{\mathcal{E}}^{\top}$ factors through $\mathrm{S}^{2} A^{*}$ and for $\mathcal{E} \simeq \mathcal{E}_{\Psi}$ it agrees with $\Psi^{\top}$. Then we may indifferently start with a general net $\Psi$ or with a general 3-instanton $\mathcal{E}$.

\section{Nets of dual quadrics and nets of alternating 2-forms}

Remark 4.1 Given a general net of dual quadrics $\Psi$ as in 3.2, the space $\operatorname{Tor}_{*}^{R}\left(R^{\Psi}, \mathbb{C}\right)_{*}$ is endowed with a natural skew-commutative algebra structure, see [27, Section 5]. In particular, we define the net of alternating 2-forms $\sigma_{\Psi}$ as the tor-multiplication $\wedge^{2} V_{\Psi} \rightarrow V_{\Psi}^{2,4}$.

By construction, see $\left[9\right.$, exercise A.3.20], the map $\sigma_{\Psi}$ fits in the commutative diagram below.

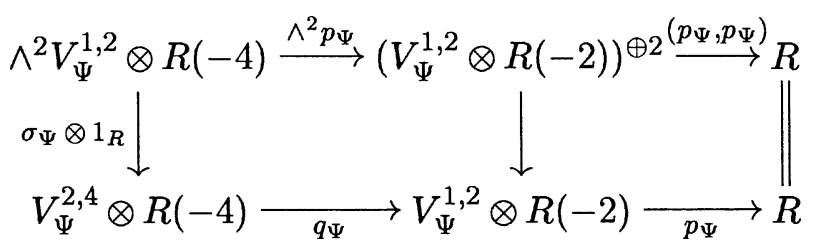

It will turn out from Theorem 4.5 that $V_{\Psi}^{2,4} \simeq B^{*}$ so we will be able to write with no ambiguity $\sigma_{\Psi}$ as a map $\wedge^{2} V_{\Psi} \rightarrow B^{*}$.

Let $V$ be a complex vector space of dimension 7 and consider the Grassmannian $\mathrm{G}=\mathbb{G}\left(\mathbb{C}^{3}, V\right)$ endowed with the rank-3 universal subbundle $U_{\mathrm{G}}$. Given a 3-dimensional complex vector space $B$, let $\sigma: \wedge^{2} V \rightarrow B^{*}$ be a net of alternating 2-forms.

Definition 4.2 Given a general net of alternating forms $\sigma: \wedge^{2} V \rightarrow B^{*}$ define

$$
X_{\sigma}=\left\{\mathbb{C}^{3} \subset V \mid \sigma^{\top}(b)(u \wedge v)=0 \text { for any } u, v \in \mathbb{C}^{3}, \text { for any } b \in B\right\}
$$

The variety $X_{\sigma}$ is a Fano threefold of type $V_{22}$ given in $\mathrm{G}$ as the zero locus of the section $\sigma$ of $B^{*} \otimes \wedge^{2}\left(U_{\mathrm{G}}^{*}\right)$. 
Lemma 4.3 (Schreyer) Given a general net dual quadrics $\Psi$ as in Definition 3.2, and the net of alternating 2-forms $\sigma_{\Psi}$ of Remark 4.1, we have an isomorphism $X_{\Psi} \simeq X_{\sigma_{\Psi}}$. Under this isomorphism $U_{\mathrm{H}}$ is taken to $U_{\mathrm{G}}$.

Remark 4.4 For a general net $\sigma$ define the map $\varsigma: V \otimes B \rightarrow V^{*}$ associated to $\sigma^{\top}: B \rightarrow \wedge^{2} V^{*}$ by $\varsigma(u \otimes b)(v)=\sigma^{\top}(b)(u \wedge v)$ for $u \in B$ and $u, v \in V$. There is an isomorphism

$$
\operatorname{Hom}_{X}\left(U_{\mathrm{G}}, Q_{\mathrm{G}}^{*}\right) \simeq B
$$

The map 5 is the transpose of the map induced on globas sections by the dual of the surjective map $p_{U, Q^{*}}: B \otimes U \rightarrow Q^{*}$.

Proof The definition of $\varsigma$ is clear. Considering the Koszul complex of $X$ in $\mathrm{G}$ one computes easily $\mathrm{H}^{0}\left(X, \mathrm{~S}^{2} U_{\mathrm{G}}^{*}\right) \simeq \mathrm{S}^{2} V^{*}$ and $\mathrm{H}^{0}\left(X, \wedge^{2} U_{\mathrm{G}}^{*}\right) \simeq \operatorname{coker} \sigma^{\top}$, obtaining $\operatorname{Hom}\left(U_{\mathrm{G}}, Q_{\mathrm{G}}^{*}\right) \simeq \mathrm{H}^{0}\left(U_{\mathrm{G}}^{*} \otimes Q_{\mathrm{G}}^{*}\right) \simeq B$. Now for $b \in B, u \in U_{\mathrm{G}}, q \in Q_{\mathrm{G}}$, we

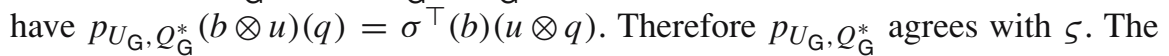
map $\varsigma$ is surjective for general $\sigma$ so $p_{U_{\mathrm{G}}, Q_{\mathrm{G}}^{*}}$ is also surjective for $U_{\mathrm{G}}^{*}$ and $Q_{\mathrm{G}}$ are globally generated.

Theorem 4.5 For a general net of dual quadrics $\Psi$ we have the following natural exact sequence

$$
0 \rightarrow Y_{\Psi} \stackrel{\ell_{1}}{\rightarrow} A \otimes V_{\Psi}^{2,3} \stackrel{\ell_{2}}{\rightarrow} B \otimes V_{\Psi} \stackrel{\ell_{3}}{\rightarrow} V_{\Psi}^{*} \rightarrow 0
$$

where the vector space $Y_{\Psi}$ and the map $\ell_{2}$ are given by

$$
\begin{array}{r}
Y_{\Psi}=\operatorname{ker}\left(\sigma_{\Psi}: \wedge^{2} V_{\Psi} \rightarrow V_{\Psi}^{2,4}\right) \\
\ell_{2}: A \otimes V_{\Psi}^{2,3} \stackrel{q_{\Psi}}{\longrightarrow} S^{2} A \otimes V_{\Psi} \stackrel{\Psi \otimes 1_{V_{\Psi}}}{\longrightarrow} B \otimes V_{\Psi}
\end{array}
$$

The map $\ell_{3}$ is defined as $\varsigma$ as in Remark 4.4 with $\sigma=\sigma_{\Psi}$. The map $\ell_{1}$ is defined lifting the inclusion $Y_{\Psi} \hookrightarrow S^{2} A \otimes V_{\Psi}$ to $A \otimes V_{\Psi}^{2,3}$ via the map $q_{\Psi}$, i.e. $\ell_{1}$ makes the following diagram commutative

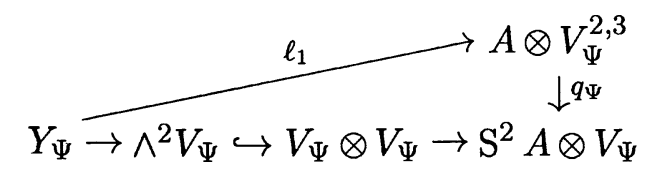

There is a canonical isomorphism $V_{\Psi}^{2,4} \simeq B^{*}$.

Proof To prove the exactness in $A \otimes V_{\Psi}^{2,3}$ we need to use the definition of $\sigma_{\Psi}$ in 4.1. In fact, considering the resolution of the ideal $J^{\Psi}$, taken in degree 4 , we write

$$
0 \rightarrow A \otimes V_{\Psi}^{2,3} \oplus V_{\Psi}^{2,4} \stackrel{q_{\Psi}}{\longrightarrow} \mathrm{S}^{2} A \otimes V_{\Psi} \rightarrow \mathrm{S}^{4} A \rightarrow 0
$$

and we need to consider the composition (10). Notice that the kernel of $\Psi \otimes 1_{V_{\Psi}}$ in $\mathrm{S}^{2} A \otimes V_{\Psi}$ is $V_{\Psi} \otimes V_{\Psi}$. Moreover it is mapped to zero by $m: \mathrm{S}^{2} A \otimes \mathrm{S}^{2} A \rightarrow$ 
$S^{4} A$, so it must lie in $\wedge^{2} S^{2} A$. Therefore we have $\operatorname{ker}\left(\Psi \otimes 1_{V_{\Psi}}\right)=\wedge^{2} S^{2} A \cap$ $V_{\Psi} \otimes V_{\Psi}=\wedge^{2} V_{\Psi}$. So we obtain the following exact sequence

$$
0 \rightarrow \wedge^{2} V_{\Psi} \rightarrow A \otimes V_{\Psi}^{2,3} \oplus V_{\Psi}^{2,4} \stackrel{\Psi \otimes 1_{V_{\Psi} \circ q_{\Psi}}}{\longrightarrow} B \otimes V_{\Psi}
$$

where the map $\ell_{1}$ of the statement is the restriction to $Y_{\Psi}$ of the above map $\wedge^{2} V_{\Psi} \rightarrow A \otimes V_{\Psi}^{2,3} \oplus V_{\Psi}^{2,4}$ and we still have to prove that $\ell_{1}$ takes image in $A \otimes V_{\Psi}^{2,3}$. Now Tor multiplication identifies the map $\sigma_{\Psi}: \wedge^{2} V_{\Psi} \rightarrow V_{\Psi}^{2,4}$ as the arrow making diagram (7) commutative. This diagram, taken in degree 4 , boils down to the following commutative diagram

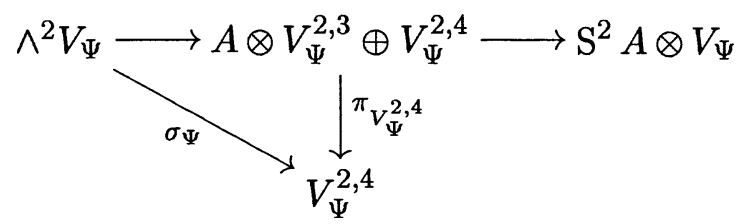

This, together with (13), proves at the same time that the map $\ell_{1}$ is well defined in $Y_{\Psi}$ and that the required sequence is exact in $A \otimes V_{\Psi}^{2,3}$. Consequently it is exact also in $Y_{\Psi}$. In order to prove exactness in $B \otimes V_{\Psi}$ we will need to use the instanton bundle $\mathcal{E}_{\Psi}$.

Denote the kernel sheaf $\operatorname{ker}\left(p_{\Psi}\right)$ by $\mathcal{K}_{\Psi}^{1}$. Taking the symmetrized powers of the sequence (5) we get

$$
\begin{aligned}
& 0 \rightarrow \mathcal{K}_{\Psi}^{2} \rightarrow S^{2} V_{\Psi} \otimes \mathcal{O} \rightarrow \mathcal{O}(4) \rightarrow 0 \\
& 0 \rightarrow \wedge^{2} \mathcal{K}_{\Psi}^{1}(4) \rightarrow V_{\Psi} \otimes \mathcal{K}_{\Psi}^{1}(2) \rightarrow \mathcal{K}_{\Psi}^{2} \rightarrow 0 \\
& 0 \rightarrow S^{2} \mathcal{K}_{\Psi}^{1}(4) \rightarrow S^{2} V_{\Psi} \otimes \mathcal{O} \rightarrow V_{\Psi} \otimes \mathcal{O}(2) \rightarrow 0 \\
& 0 \rightarrow \wedge^{2} \mathcal{K}_{\Psi}^{1}(4) \rightarrow \wedge^{2} V_{\Psi} \otimes \mathcal{O} \rightarrow \mathcal{K}_{\Psi}^{1}(4) \rightarrow 0
\end{aligned}
$$

for some vector bundle $\mathcal{K}_{\Psi}^{2}$. In turn the symmetrized square of the sequence (6) gives the following

$$
\begin{aligned}
& 0 \rightarrow \mathcal{O}(-6) \rightarrow \wedge^{2} V_{\Psi}^{2,3} \otimes \mathcal{O}(-2) \rightarrow \mathcal{K}_{\Psi}^{3} \rightarrow 0 \\
& 0 \rightarrow \mathcal{K}_{\Psi}^{3} \rightarrow V_{\Psi}^{2,3} \otimes \mathcal{K}_{\Psi}^{1}(1) \rightarrow S^{2} \mathcal{K}_{\Psi}^{1}(4) \rightarrow 0
\end{aligned}
$$

for some vector bundle $\mathcal{K}_{\Psi}^{3}$. So we get the the following commutative diagram with exact rows and columns.

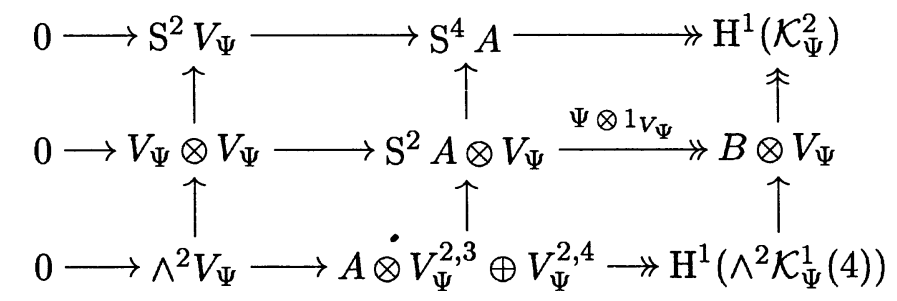

Here the left vertical column is the canonical decomposition $V_{\Psi}^{\otimes 2}$ into symmetric and skew-symmetric tensors, the central vertical row is (12) and the bottom 
row is the cohomology sequence of (17). This yields two presentations of $\mathrm{H}^{1}\left(\mathcal{K}_{\Psi}^{2}\right)$ (the vertical one from (15) and the horizontal one from (14)).

On the other hand $\mathcal{K}_{\Psi}^{2}$ defined in (14) and $\mathrm{S}^{2} \mathcal{K}_{\Psi}^{1}$ (4) fit into the following short exact sequence

$$
0 \rightarrow S^{2} \mathcal{K}_{\Psi}^{1}(4) \rightarrow \mathcal{K}_{\Psi}^{2} \rightarrow \mathcal{K}_{\Psi}^{1}(4) \rightarrow 0
$$

Summing up we can then build the following commutative diagram (we omit surrounding zeroes for brevity)

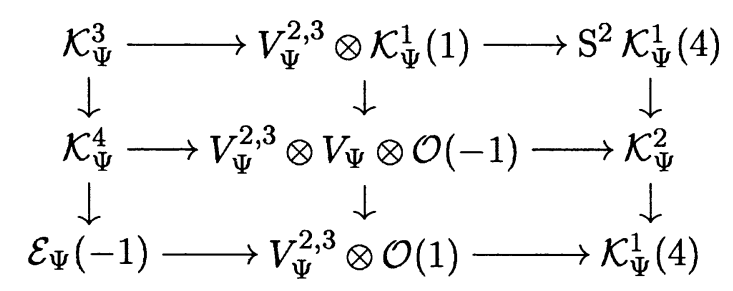

where the top (respectively bottom) horizontal row is (19) (respectively 6). The central row defines some bundle $\mathcal{K}_{\Psi}^{4}$ as the kernel of the composition of the two projections $V_{\Psi}^{2,3} \otimes V_{\Psi} \otimes \mathcal{O}(-1) \rightarrow V_{\Psi} \otimes \mathcal{K}_{\Psi}^{1}(2)$ and $V_{\Psi} \otimes \mathcal{K}_{\Psi}^{1}(2) \rightarrow \mathcal{K}_{\Psi}^{2}$ of (6) and (15). The right (respectively central) vertical column comes from (20) (respectively comes from (5)). Use (18) to show $\mathrm{H}^{2}\left(\mathcal{K}_{\Psi}^{3}\right) \simeq \mathrm{S}^{2} A^{*}$ and Lemma 3.5 for $\mathrm{H}^{1}\left(\mathcal{E}_{\Psi}(-1)\right) \simeq B^{*}$. Now taking cohomology in the diagram (21) we get

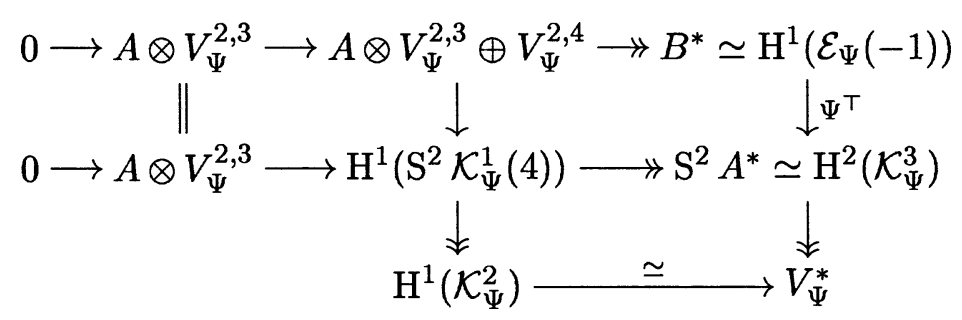

This provides the following isomorphisms

$$
\begin{aligned}
& V_{\Psi}^{2,4} \simeq B^{*} \\
& V_{\Psi}^{*} \simeq \operatorname{coker}\left(\mathrm{S}^{2} V_{\Psi} \rightarrow \mathrm{S}^{4} A\right) \simeq \mathrm{H}^{1}\left(\mathcal{K}_{\Psi}^{2}\right) \\
& V_{\Psi}^{*} \simeq \operatorname{coker}\left(A \otimes V_{\Psi}^{2,3} \rightarrow B \otimes V_{\Psi}\right) \simeq \mathrm{H}^{1}\left(\mathcal{K}_{\Psi}^{2}\right)
\end{aligned}
$$

Thus we proved the exactness of the sequence (8).

\section{Polar hexagons to a plane quartic}

5.1 The variety of sums of powers

Let $B$ be a 3-dimensional $\mathbb{C}$-vector space and $f \in \mathrm{S}^{4} B$ be a plane quartic. Put $\breve{\mathbb{P}}^{2}=\mathbb{P}\left(B^{*}\right)$. According to Mukai [21], we define the subvariety of $\operatorname{Hilb}_{6}\left(\breve{\mathbb{P}}^{2}\right)$ consisting of polar hexagons to $f$. 
Definition 5.1 Given a general quartic form $f \in \mathrm{S}^{4} B=\mathrm{H}^{0}\left(\mathbb{P}^{2}, \mathcal{O}(4)\right)$ define the variety of sums of powers as

$$
\operatorname{VSP}(6, f)=\overline{\left\{f_{1}, \ldots, f_{6} \mid f_{1}^{4}+\ldots+f_{6}^{4}=f\right\}}
$$

where the closure is taken in $\mathrm{Hilb}_{6}\left(\check{\mathbb{P}}^{2}\right)$.

Lemma 5.2 (Mukai, Schreyer) For general $f$ the variety $\operatorname{VSP}(6, f)$ is a Fano threefold of type $V_{22}$. Given a net of dual quadrics $\Psi$ as in Definition 3.2, there exists a quartic such that $\operatorname{VSP}(6, f) \simeq X_{\Psi}$.

Remark 5.3 Considering the apolarity action of $T^{*}$ on $T$ (cfr. Sect. 3) we may view $f$ as the map $f: B^{*} \rightarrow S^{3} B$ taking $\partial$ to $\partial(f)$. This map is injective for general $f$ so we can define $V_{f}=S^{3} B / f\left(B^{*}\right)$. Under the hypothesis of Lemma 5.2, there is a natural isomorphism $V_{\Psi} \simeq \mathrm{S}^{3} B / f\left(B^{*}\right)$.

Definition 5.4 Let $f$ be a general plane quartic and let $X=\operatorname{VSP}(6, f)$. Then there is a rank-3 vector bundle $U_{\mathrm{VSP}}$ (respectively a rank-5 vector bundle $K_{\mathrm{VSP}}$ ) on $\operatorname{VSP}(6, f)$, whose fiber over an element $\Lambda=\left(f_{1}, \ldots, f_{6}\right) \in \operatorname{VSP}(6, f)$ is $\left\langle f_{1}^{3}, \ldots, f_{6}^{3}\right\rangle / f\left(B^{*}\right)$ (respectively the fiber is $\left.\left\langle f_{1}^{4}, \ldots, f_{6}^{4}\right\rangle / f\right)$. This bundle embeds $X$ into $\mathbb{G}\left(\mathbb{C}^{3}, V_{f}\right)$ (respectively into $\mathbb{G}\left(\mathbb{C}^{3}, \mathrm{~S}^{4} B / f\right)$ ) (see Remark 5.3). Denote by $Q_{\mathrm{VSP}}^{*}$ the restriction to $X$ of the universal rank-4 quotient bundle on $\mathbb{G}\left(\mathbb{C}^{3}, V_{f}\right)$.

\subsection{The Hilbert scheme}

For any $\Lambda \in \operatorname{Hilb}_{6}\left(\check{\mathbb{P}}^{2}\right)$ we can consider the resolution of the ideal $J_{\Lambda, \check{\mathbb{P}}^{2}}$ over the ring $T^{*}=\mathbb{C}\left[B^{*}\right]$. For a general length-6 subscheme $\Lambda$ the resolution reads

$$
0 \leftarrow J_{\Lambda, \breve{\mathbb{P}}^{2}} \leftarrow T^{*}(-3)^{4} \leftarrow T^{*}(-4)^{3} \leftarrow 0
$$

The resolution has this shape whenever no conic of $\check{\mathbb{P}}^{2}$ passes through $\Lambda$ and no line cuts a length-3 subscheme of $\Lambda$. This open set, which we denote by Hilb $_{6}\left(\check{\mathbb{P}}^{2}\right)^{\circ}$, embeds into $\mathbb{G}\left(\mathbb{C}^{4}, \mathrm{~S}^{3} B^{*}\right)$ by means of a rank-4 vector bundle $Q_{\mathrm{L}}^{*}$. The fiber of $Q_{\mathrm{L}}^{*}$ over $\Lambda$ defined as $\operatorname{Tor}_{1}^{T^{*}}\left(T^{*} / J_{\Lambda, \check{\mathbb{P}}^{2}}, \mathbb{C}\right)_{3} \simeq \mathbb{C}^{4}$ i.e. we take the space of cubics vanishing on $\Lambda$. We have $\mathrm{H}^{0}\left(Q_{\mathrm{L}}\right)^{*} \simeq \mathrm{S}^{3} B^{*}$.

Moreover we have a rank-3 vector bundle $U_{\mathrm{L}}$ on $\mathrm{Hilb}_{6}\left(\check{\mathbb{P}}^{2}\right)^{\circ}$ whose fiber over $\Lambda$ is the 3 -space of first-order syzygies of $\Lambda$. Equivalently we take $\operatorname{Tor}_{2}^{T^{*}}\left(T^{*} / J_{\Lambda, \check{\mathbb{P}}_{2}}, \mathbb{C}\right)_{4} \simeq \mathbb{C}^{3}$. We have $\mathrm{H}^{0}\left(U_{\mathrm{L}}^{*}\right)^{*} \simeq \mathrm{S}^{3,1} B^{*} \simeq$ $\operatorname{ker}\left(\mathrm{S}^{3} B^{*} \otimes B^{*} \rightarrow \mathrm{S}^{4} B^{*}\right)$. One computes $\operatorname{dim}\left(\mathrm{S}^{3,1} B^{*}\right)=15$. The bundle $U_{\mathrm{L}}^{*}$ provides an embedding Hilb $6\left(\check{\mathbb{P}}^{2}\right)^{\circ} \subset \mathbb{G}\left(\mathbb{C}^{3}, \mathrm{~S}^{3,1} B^{*}\right)$. The following Lemma is proved in [27, Theorem 2.3] except for the last statement that follows from [27, Theorem 2.6]. 
Lemma 5.5 (Schreyer) Let $f \in \mathrm{S}^{4} B$ be a general quartic and, making use of the apolarity pairing (cfr Sect. 3), define the ideal

$$
J^{f}=\left\{s \in T^{*} \mid s(f)=0\right\}
$$

Then the ring $\left(T^{*}\right)^{f}=T^{*} / J^{f}$ is Artinian Gorenstein and its minimal graded free resolution over $T^{*}$ takes the form

$$
0 \leftarrow\left(T^{*}\right)^{f} \leftarrow T^{*} \leftarrow V_{f}^{1,3} \otimes T^{*}(-3) \leftarrow V_{f}^{2,4} \otimes T^{*}(-4) \leftarrow T^{*}(-7) \leftarrow 0
$$

where $V_{f}^{i, j}=\operatorname{Tor}_{i}^{T^{*}}\left(\left(T^{*}\right)^{f}, \mathbb{C}\right)_{j}$. We have $\operatorname{dim}\left(\mathrm{V}_{f}^{1,3}\right)=\operatorname{dim}\left(\mathrm{V}_{f}^{2,4}\right)=7$ and there is a canonical duality $\left(V_{f}^{1,3}\right)^{*} \simeq V_{f}^{2,4}$.

The map $\varsigma_{f}^{\top}: V_{f}^{2,4} \rightarrow B^{*} \otimes V_{f}^{1,3}$ defined by (22) is skew-symmetric and induces $\sigma_{f}^{\top}: B \rightarrow \wedge^{2}\left(V_{f}^{2,4}\right)^{*}\left(\right.$ cfr. Remark 4.4). We have $X_{\sigma_{f}} \simeq \operatorname{VSP}(6, f)$ (see Definitions 4.2 and 5.1).

Thus, the rank-4 bundle $Q_{\mathrm{L}}$ provides an embedding $X \hookrightarrow \mathbb{G}\left(\mathbb{C}^{4}, V_{f}^{1,3}\right)$ while the rank-3 bundle $U_{\mathrm{L}}^{*}$ gives $X \hookrightarrow \mathbb{G}\left(\mathbb{C}^{4}, V_{f}^{2,4}\right)$ where $V_{f}^{1,3}$ and $V_{f}^{2,4}$ are dual 7-dimensional complex vector spaces.

Remark 5.6 After restriction to $X=\operatorname{VSP}(6, f)$ there are natural isomorphisms $Q_{\mathrm{VSP}}^{*} \simeq Q_{\mathrm{L}}^{*}$ and $U_{\mathrm{VSP}} \simeq U_{\mathrm{L}}$. On $X=\operatorname{VSP}(6, f)$ we have $\mathrm{H}^{0}\left(Q_{\mathrm{L}}\right)^{*} \simeq V_{f}^{1,3} \simeq$ $\operatorname{ker}\left(f^{\top}: \mathrm{S}^{3} B^{*} \rightarrow B\right), \mathrm{H}^{0}\left(U_{\mathrm{L}}^{*}\right)^{*} \simeq V_{f}^{2,4} \simeq V_{f}$.

Proof The isomorphism $V_{f}^{1,3} \simeq \operatorname{ker}\left(f^{\top}: \mathrm{S}^{3} B^{*} \rightarrow B\right)$ is clear, indeed, by the definition of $J_{f}$, the cubic forms that generate $J^{f}$ (i.e. the space $V_{f}^{1,3}$ by Lemma 5.5) are those annihilated by $f^{\top}: \mathrm{S}^{3} B^{*} \rightarrow B$ under the apolarity pairing (i.e. the space $\operatorname{ker}\left(f^{\top}\right)$ ). Also, we have $\mathrm{H}^{0}\left(Q_{\mathrm{L}}\right)^{*} \simeq V_{f}^{1,3}$ since by Lemma 5.5 the cubic forms vanishing on a length- 6 subschema $\Lambda$ with $[\Lambda] \in \operatorname{VSP}(6, f)$ lie in $V_{f}^{1,3}$.

Now, given $\Lambda=\left(f_{1}, \ldots, f_{6}\right) \in \operatorname{VSP}(6, f)$, the fiber of $Q_{\mathrm{L}, \Lambda}^{*}$ consists of those elements in $S^{3} B^{*}$ (and actually in $V_{f}^{1,3} \subset S^{3} B^{*}$ ) that vanish identically on $\Lambda$, i.e. that annihilate $f_{1}^{3}, \ldots, f_{6}^{3}$ under the apolarity pairing (cfr. Remark 5.3). Equivalently we take the degree-3 generators of the ideal $J_{\Lambda, \check{\mathbb{P}}^{2}}$, i.e. $Q_{\mathrm{VSP}, \Lambda}^{*}$. Thus we have $Q_{\mathrm{L}, \Lambda}^{*} \simeq Q_{\mathrm{VSP}, \Lambda}^{*}$.

Then we also have $U_{\mathrm{L}} \simeq U_{\mathrm{VSP}}$ since they are both isomorphic to ker $p_{\mathcal{O}, Q_{\mathrm{VSP}}}$. By virtue of the duality in Lemma 5.5, we also have $\mathrm{H}^{0}\left(U^{*}\right)^{*} \simeq V_{f}^{2,4} \simeq$ $\left(V^{1,3}\right)_{f}^{*} \simeq V_{f}$.

\subsection{The variety of Kronecker modules}

Following Drezet we introduce the following variety of Kronecker modules. Consider the space of $3 \times 4$ matrices with entries in $B^{*}$ and the G.I.T. quotient

$$
\mathrm{K}=\mathrm{M}_{3 \times 4}\left(B^{*}\right) / / \mathrm{SL}(3) \times \mathrm{SL}(4)
$$


An element $[\gamma] \in \mathrm{K}$ is represented by $\gamma: V_{s(\gamma)} \rightarrow V_{t(\gamma)} \otimes B^{*}$ where $V_{s(\gamma)} \simeq$ $\mathbb{C}^{3}$ and $V_{t(\gamma)} \simeq \mathbb{C}^{4}$ denote the source and target vector spaces of the map $\gamma$. The variety $\mathrm{K}$ has been studied in detail in [8]. It is endowed with two natural bundles, $Q_{\mathrm{K}}^{*}$ (respectively $U_{\mathrm{K}}$ ) of rank 4 (respectively 3 ), whose fiber over $[\gamma] \in \mathrm{K}$ is $V_{s(\gamma)}$ (respectively $\left.V_{t(\gamma)}\right)$. The bundles $U_{\mathrm{K}}$ and $Q_{\mathrm{K}}^{*}$ are related by

$$
\operatorname{Hom}_{\mathrm{K}}\left(U_{\mathrm{K}}, Q_{\mathrm{K}}^{*}\right) \simeq B
$$

Lemma 5.7 (Drezet) There is a birational map $\delta: \mathrm{Hilb}_{6}\left(\breve{\mathbb{P}}^{2}\right) \rightarrow \mathrm{K} d e$ fined over Hilb $6\left(\check{\mathbb{P}}^{2}\right)^{\circ}$ associating to $\Lambda$ the map $\gamma: \operatorname{Tor}_{2}^{T^{*}}\left(T^{*} / J_{\Lambda, \check{\mathbb{P}}^{2}}, \mathbb{C}\right)_{4} \rightarrow$ $B^{*} \otimes \operatorname{Tor}_{1}^{T^{*}}\left(T^{*} / J_{\Lambda, \breve{\mathbb{P}} 2}, \mathbb{C}\right)_{3}$. Denote by $\mathrm{K}^{\circ}$ the open subset of $\mathrm{K}$ isomorphic via $\delta$ to $\mathrm{Hilb}_{6}\left(\check{\mathbb{P}}^{2}\right)^{\circ}$.

Notice that under the isomorphism $\delta_{\mid \mathrm{Hilb}_{6}\left(\check{\mathbb{P}}^{2}\right)^{\circ}}$, the bundle $U_{\mathrm{K}}$ is pulled back to $U_{\mathrm{L}}$ and $Q_{\mathrm{K}}^{*}$ is pulled back to $Q_{\mathrm{L}}^{*}$.

Lemma 5.8 Define $P_{\mathrm{K}}$ as $P_{\mathrm{K}}=\operatorname{coker}\left(i_{U_{\mathrm{K}}, Q_{\mathrm{K}}^{*}}: U_{\mathrm{K}} \rightarrow B^{*} \otimes Q_{\mathrm{K}}^{*}\right)$. Then $P_{\mathrm{K}}$ is locally free of rank 9 over $\mathrm{K}^{\circ}$. The fiber of $P_{\mathrm{K}}$ is identified with $\mathrm{H}^{0}\left(J_{\Lambda, \check{\mathbb{P}}^{2}}(4)\right) / f$.

The bundle $P_{\mathrm{K}}^{*}$ is globally generated with $\mathrm{H}^{0}\left(P_{\mathrm{K}}^{*}\right) \simeq \mathrm{S}^{4} B$. The zero locus in $\mathbb{G}\left(3 \times 4, B^{*}\right)$ of its general section $f$ is a Fano threefold of type $V_{22}$ of the form $\operatorname{VSP}(6, f)$ defined in 5.1.

Proof The bundle $P_{\mathrm{K}}^{*}$ is globally generated since $Q_{\mathrm{K}}$ and $U_{\mathrm{K}}^{*}$ are. Recall that $\mathrm{H}^{0}\left(Q_{\mathrm{L}}\right)^{*} \simeq \mathrm{S}^{3} B^{*}$ and $\mathrm{H}^{0}\left(U_{\mathrm{L}}^{*}\right)^{*} \simeq \mathrm{S}^{3,1} B^{*}$. Computing global sections of $P_{\mathrm{K}}^{*}$ via the map $\delta$ defined in 5.7 we get the $\mathrm{H}^{0}\left(P_{\mathrm{K}}^{*}\right) \simeq \mathrm{S}^{4} B$. Now the condition for a point $\Lambda \in \operatorname{Hilb}_{6}\left(\check{\mathbb{P}}^{2}\right)$ to lie in $X$ is that the generators of its ideal, as elements of $S^{3} B^{*}$, multiplied by any linear form $\partial \in B^{*}$, map to zero under the evaluation with $f \in\left(\mathrm{S}^{4} B^{*}\right)^{*} \simeq \mathrm{S}^{4} B$.

This means that $\Lambda$ lies in the zero locus of the section $f$ of the kernel bundle $P_{\mathrm{K}}^{*}$, since the map induced on global sections by the evaluation $B \otimes Q_{\mathrm{K}} \rightarrow U_{\mathrm{K}}^{*}$ is just the multiplication $m$ in $T$. So the zero locus of a section $f$ of $P_{\mathrm{K}}^{*}$ is isomorphic to the variety $\operatorname{VSP}(6, f)$.

Remark 5.9 In the framework of Lemma 5.8, there exists a rank-5 bundle $K_{\mathrm{K}}=$ $\operatorname{ker}\left(p_{\mathcal{O}, P_{\mathrm{K}}^{*}}: \mathrm{H}^{0}\left(P_{\mathrm{K}}^{*}\right) \otimes \mathcal{O}_{\mathrm{K}} \rightarrow P_{\mathrm{K}}^{*}\right)$. Under the identifications of Remark 5.6 we have an isomorphism on $X, K_{\mathrm{VSP}} \simeq K_{\mathrm{K}}$.

Proof It is easy to show that there exists the following commutative diagram with exact rows and columns (we omit zeroes surrounding all the diagram for brevity).

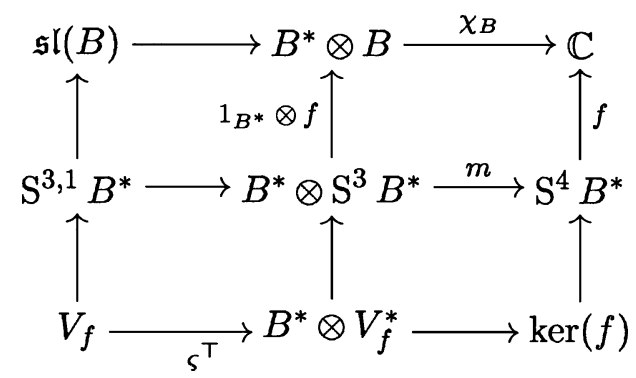


Here $f$ is considered alternatively as a map $\mathrm{S}^{3} B^{*} \rightarrow B$ (in the central column) or as an element of $\mathrm{S}^{4} B \simeq\left(\mathrm{S}^{4} B^{*}\right)^{*}$ (in the right column) and the bottom row is defined by Remark 4.4. Since $K_{\mathrm{VSP}}^{*}$ is globally generated with $\mathrm{H}^{0}\left(K_{\mathrm{VSP}}^{*}\right)^{*} \simeq$ ker $f \subset S^{4} B^{*}$ by Definition 5.4, the bottom row of (23) proves the following

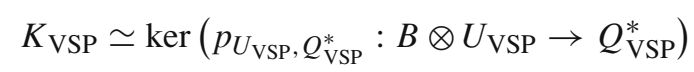

On the other hand by definition of $K_{\mathrm{K}}$ we have

$$
K_{\mathrm{K}} \simeq \operatorname{ker}\left(p_{U_{\mathrm{K}}, Q_{\mathrm{K}}^{*}}: B \otimes U_{\mathrm{K}} \rightarrow Q_{\mathrm{K}}^{*}\right)
$$

Thus we conclude keeping in mind Lemma 5.7.

\section{Bundles on $X$}

Throughout the rest of the paper, $X$ will be a Fano threefold of type $V_{22}$ defined by a general net of dual quadrics $\Psi$ as $X=X_{\Psi}$ according to Definition 3.2. In particular, we fix a 3-dimensional (respectively, 4-dimensional) $\mathbb{C}$-vector space $B$ (respectively $A$ ). We will keep in mind the isomorphisms of Lemmas 4.3, 5.2 and of Remarks 5.3, 5.6 and 5.9.

Then, we denote by $U$ (respectively $Q^{*}$ and $K$ ) the rank-3 (respectively rank-4 and rank-5) bundles on $X$ defined by any of the constructions of Sects. (3), (4) and (5). We will often drop the subscript $\Psi$, e.g. $V_{\Psi}^{2,3}=V^{2,3}$ and we will write $E$ for the bundle $E_{\mathrm{H}}$ restricted to $X$.

Lemma 6.1 There are the following natural isomorphisms

$$
\operatorname{Hom}\left(U, Q^{*}\right) \simeq B \quad \operatorname{Hom}(E, U) \simeq A^{*} \quad \operatorname{Hom}(K, U) \simeq B^{*}
$$

Furthermore there are the following natural exact sequences

$$
\begin{aligned}
& 0 \rightarrow K \rightarrow B \otimes U \rightarrow Q^{*} \rightarrow 0 \\
& 0 \rightarrow U \rightarrow B^{*} \otimes Q^{*} \rightarrow P \rightarrow 0 \\
& 0 \rightarrow K \rightarrow \mathrm{H}^{0}\left(K^{*}\right)^{*} \otimes \mathcal{O} \rightarrow P^{*} \rightarrow 0 \\
& 0 \rightarrow U \rightarrow V \otimes \mathcal{O} \rightarrow Q^{*} \rightarrow 0
\end{aligned}
$$

Finally we have the following Chern classes

$$
\begin{array}{lll}
c_{1}(U)=-1 & c_{2}(U)=10 & c_{3}(U)=-2 \\
c_{1}\left(Q^{*}\right)=-1 & c_{2}\left(Q^{*}\right)=12 & c_{3}\left(Q^{*}\right)=-4 \\
c_{1}(K)=-2 & c_{2}(K)=40 & c_{3}(K)=-20 \\
c_{1}(P)=-2 & c_{2}(P)=48 & c_{3}(P)=-36
\end{array}
$$

Proof It is straightforward to compute the Chern classes of the bundles involved in our statement. Further, the isomorphisms in (26) follow the from Remarks 5.9, 4.4 and Lemma 3.1 by restriction to $X$, as well as the exact sequences (27). The exact sequence (28) follows from Lemma 5.8. Combining (27) and (28) one obtains the following commutative diagram with exact rows and columns (we omit surrounding zeroes for brevity) 


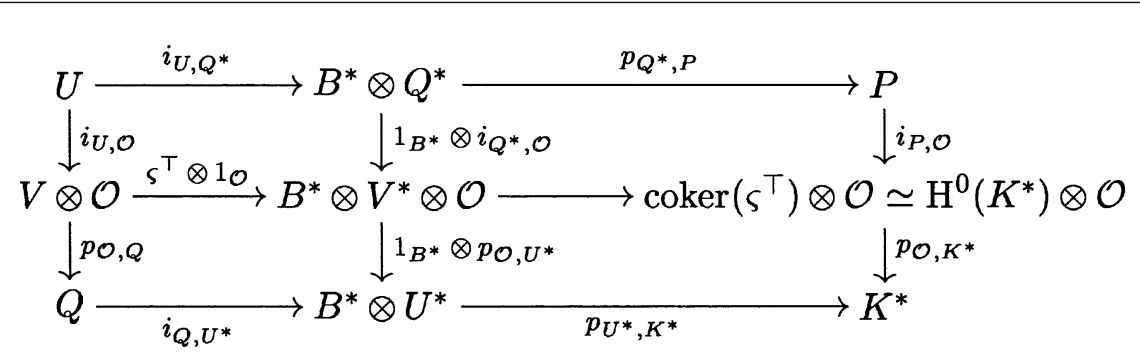

where $\varsigma$ is defined in Remark 4.4 and the vertical arrows in the first two columns are the obvious ones. Then the last column yields the exact sequence (29).

Lemma 6.2 The bundles $U, Q, K$ are aCM stable sheaves on $X$.

Proof It follows from Definition 4.2 that $X$ is the zero locus in $\mathbb{G}\left(\mathbb{C}^{3}, V\right)$ of a section of the globally generated bundle $U(1)^{3}$. Taking the Koszul complex associated to this section tensorized by $U(t)$ and using Bott theorem (see [5]) over $\mathbb{G}\left(\mathbb{C}^{3}, V\right)$ one computes the required vanishing for $U$ and $Q$ in order to show that they are aCM sheaves. Using the exact sequence (27) it is immediate to show that $K$ is also an aCM sheaf.

Since $c_{1}(U)=c_{1}\left(Q^{*}\right)=-1$ and $\mathrm{h}^{0}(U)=\mathrm{h}^{0}\left(Q^{*}\right)=0$, and since $U^{*}$ and $Q$ are globally generated, it follows easily from Lemma 2.4 that $U$ and $Q$ are stable. From the exact sequence (27) one sees that $\mathrm{h}^{0}(K)=\mathrm{h}^{0}\left(\wedge^{2} K\right)=0$. Finally from $\wedge^{p} K(1) \simeq \wedge^{5-p} K^{*}(-1)$ and again using (27) it follows that $\mathrm{h}^{0}\left(\left(\wedge^{3} K\right)_{n}\right)=$ $\mathrm{h}^{0}\left(\left(\wedge^{4} K\right)_{n}\right)=0$. Thus we conclude by Lemma 2.4.

Lemma 6.3 The bundle $E^{*}$ is globally generated with $\mathrm{H}^{0}\left(E^{*}\right)^{*} \simeq V^{2,3} \simeq \mathbb{C}^{8}$. There is a rank- 6 bundle $L$ defined by the exact sequence

$$
0 \rightarrow E \rightarrow V^{2,3} \otimes \mathcal{O} \rightarrow L^{*} \rightarrow 0
$$

There exists a rank-10 vector bundle $M$ with $\mathrm{H}^{0}\left(M^{*}\right)^{*} \simeq \mathrm{S}^{3} A$, whose fiber over $[\Gamma] \in X=X_{\Psi}$ is $\mathrm{H}^{0}\left(J_{\Gamma, \mathbb{P}(A)}(3)\right) \simeq \mathbb{C}^{10}$ according to Definition 3.2. There are the exact sequences

$$
\begin{aligned}
& 0 \rightarrow E \stackrel{i_{E, U}}{\rightarrow} A \otimes U \stackrel{p_{U, M}}{\rightarrow} M \rightarrow 0 \\
& 0 \rightarrow V^{2,3} \rightarrow A \otimes V \stackrel{m}{\rightarrow} S^{3} A \rightarrow 0
\end{aligned}
$$

where (33) is obtained dualizing global sections of the dual of (32) and $m$ is the composition of the obvious maps $A \otimes V \hookrightarrow A \otimes S^{2} A \rightarrow S^{3} A$.

Finally, $M^{*}$ is globally generated and there exists a rank-10 vector bundle $N$ defined by the exact sequence

$$
0 \rightarrow M \rightarrow \mathrm{S}^{3} A \otimes \mathcal{O} \rightarrow N^{*} \rightarrow 0
$$

Proof By the discussion in Sect. 3 and Definition 3.4, the fiber of the bundle $E$ over any point of $X$ embeds in $V_{\Psi}^{2,3} \simeq \mathbb{C}^{8}$, so $\mathrm{H}^{0}\left(E^{*}\right)^{*} \simeq V_{\Psi}^{2,3}$ and $E^{*}$ is globally generated and we have the exact sequence (31).

The map $E \rightarrow A \otimes U$ in (32) is obtained globalizing $q_{\Psi}$ in the resolution (4). Equivalently over any $[\Gamma] \in \mathrm{H}_{c}$ we take the linear map $A \otimes \operatorname{Tor}_{2}\left(R / J_{\Gamma, \mathbb{P}^{3}}, \mathbb{C}\right)_{3} \rightarrow$ 
$\operatorname{Tor}_{1}\left(R / J_{\Gamma, \mathbb{P}^{3}}, \mathbb{C}\right)_{2}$ given by the $2 \times 3$ matrix of linear forms whose $2 \times 2$ minors define $\Gamma$. Therefore (33) follows at once from (4). 10.

Finally, it is clear that $M^{*}$ is globally generated and $\operatorname{rk}(N)=\operatorname{dim}\left(\mathrm{S}^{3} A\right)-10=$

Proposition 6.4 We have $\operatorname{Hom}\left(\wedge^{2} U, E\right) \simeq A^{*}$ and we define the following maps (cfr. Lemma 6.1)

$$
\begin{aligned}
& e_{1}=i_{\wedge^{2} U, E}: \wedge^{2} U \rightarrow A \otimes E \\
& e_{2}=\Psi \circ i_{E, U}: A \otimes E \rightarrow B \otimes U \\
& e_{3}=p_{U, Q^{*}}: B \otimes U \rightarrow Q^{*}
\end{aligned}
$$

Then the following sequence is exact

$$
0 \rightarrow \wedge^{2} U \stackrel{e_{1}}{\rightarrow} A \otimes E \stackrel{e_{2}}{\rightarrow} B \otimes U \stackrel{e_{3}}{\rightarrow} Q^{*} \rightarrow 0
$$

Proof The dual of all the bundles appearing in the sequence (38) are globally generated, hence the sequence is exact if we prove that transpose of the maps $e_{1}$, $e_{2}$ and $e_{3}$ induce an exact sequence on global sections of the dual bundles. Denote these maps by $\overline{e_{j}}$ for $j=1,2,3$.

It is clear that ${\overline{e_{3}}}^{\top}=\ell_{3}$ of Theorem 4.5. Since $i_{E, U}$ maps the syzygy of a twisted cubic $\Gamma$ with $[\Gamma] \in X$ to the $2 \times 3$ matrix of linear forms in the minimal graded free resolution of $J_{\Gamma, \mathbb{P}^{3}}$ (see Lemma 6.3), the map on global section $A \otimes \mathrm{H}^{0}\left(E^{*}\right)^{*} \rightarrow \mathrm{S}^{2} A \otimes \mathrm{H}^{0}\left(U^{*}\right)^{*}$ induced by $i_{E, U}$ agrees with $q_{\Psi}$. Therefore we have ${\overline{e_{2}}}^{\top}=\ell_{2}$.

Now recall that $\operatorname{Hom}\left(\wedge^{2} U, E\right) \simeq \operatorname{Hom}\left(E^{*}, \wedge^{2} U^{*}\right) \simeq \operatorname{Hom}(E, U) \simeq A^{*}(\mathrm{cfr}$. Lemma 6.1 and Lemma 3.1), thus we have the map $i_{\wedge^{2} U, E}: \wedge^{2} U \rightarrow A \otimes E$. Since $\sigma=\sigma_{\Psi}$ by Definition 4.2 and Lemma 4.3 we have $\mathrm{H}^{0}\left(\wedge^{2} U^{*}\right)^{*} \simeq \operatorname{ker}(\sigma$ : $\left.\wedge^{2} V \rightarrow B^{*}\right) \simeq Y_{\Psi}$. Thus we have the map ${\overline{e_{1}}}^{\top}: Y_{\Psi} \rightarrow A \otimes V_{\Psi}^{2,3}$, and we need to prove that is coincides with $\ell_{1}$. Observe that the map $i_{\wedge^{2} U, E}$ is defined by restriction from $\mathrm{H}$ of the map $i_{\wedge^{2} U_{\mathrm{H}}, E_{\mathrm{H}}}$. Now by Lemma $3.1 i_{\wedge}^{2} U, E$ is induced by the diagram (1). On the other hand by Theorem $4.5 i_{\wedge^{2} U, E}$ is induced by the diagram (11). Since the diagram (11) is obtained restricting to $X$ the global sections spaces appearing in the diagram (1), we have ${\overline{e_{1}}}^{\top}=\ell_{1}$. Thus we conclude by Theorem 4.5 .

Remark 6.5 We can define a map $\bar{\psi}: A \rightarrow A^{*} \otimes B$ associated to the net $\Psi^{\top}$ : $B^{*} \rightarrow S^{2} A^{*}$. Indeed we put $\bar{\psi}(a)(b \otimes \partial)=\Psi(\partial(a \otimes b))$ for $a, b \in A$ and $\partial \in B^{*}$. In turn we have a map

$$
\psi: A \otimes U \rightarrow A^{*} \otimes Q^{*}
$$

and the map $\psi^{\top}: A \otimes Q \rightarrow A^{*} \otimes U^{*}$ is defined by the formula $\psi^{\top}(a \otimes q)(b \otimes u)$ $=\Psi(a \otimes b)(u)(q)$ under the identification $B \simeq \operatorname{Hom}\left(U, Q^{*}\right)$.

Lemma 6.6 Given a general net of dual quadrics $\Psi: A \otimes A \rightarrow B$, using $\sigma=\sigma_{\Psi}$ of Remark 4.1 define a map $\kappa: A \otimes V \rightarrow A^{*} \otimes V^{*}$ by

$$
\kappa(a \otimes u)(b \otimes v)=\sigma^{\top}(\Psi(a \otimes b))(u \otimes v)
$$


The map $\kappa$ is induced on global sections by $\psi$. There is an exact sequence

$$
0 \rightarrow V^{2,3} \stackrel{q_{\Psi}}{\rightarrow} A \otimes V \stackrel{\kappa}{\rightarrow} A^{*} \otimes V^{*} \stackrel{q_{\Psi}^{\top}}{\longrightarrow}\left(V^{2,3}\right)^{*} \rightarrow 0
$$

Furthermore, there is a skew-symmetric duality $\bar{\kappa}: \mathrm{S}^{3} A \rightarrow \mathrm{S}^{3} A^{*}$ such that the following diagram is commutative

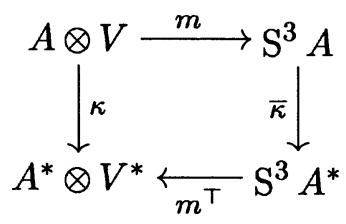

Proof The definition of $\kappa$ is clear and implies $\kappa(a \otimes u)(b \otimes v)=\kappa(b \otimes u)(a \otimes v)$ $=-\kappa(b \otimes v)(a \otimes u)$. Since $\kappa^{\top}(a \otimes u)(b \otimes v)=\kappa(b \otimes v)(a \otimes u)$, we have that $\kappa$ is skew-symmetric.

Taking the minimal graded free resolution (4) of $R^{\Psi}$ in degree 3 and 4 we get the exact sequences (33) and (12), and we denote, for the sake of this proof, by $q_{\psi}^{3}$ (respectively by $q_{\psi}^{4}$ ) the map $q_{\Psi}$ in degree 3 (respectively in degree 4 ).

Now, by the exact sequence (8) we have $\sum \alpha_{i, j} \sigma^{\top}\left(\Psi\left(a_{i} \otimes b\right)\right)\left(u \otimes v_{j}\right)=$ 0 if $\sum \alpha_{i, j} b \otimes a_{i} \otimes v_{j} \in \operatorname{Im}\left(q_{\Psi}^{4}\right)$, for some coefficients $\alpha_{i, j}$. Then $\sum \alpha_{i, j} \sigma^{\top}\left(\Psi\left(a_{i} \otimes b\right)\right)\left(u \otimes v_{j}\right)=0$ if $\sum \alpha_{i, j} a_{i} \otimes v_{j} \in \operatorname{Im}\left(q_{\Psi}^{3}\right)$. Thus there exists a map $\phi: S^{3} A \rightarrow A^{*} \otimes V^{*}$ with $\phi \circ m=\kappa$. On the other hand, again by (8), if $\sum \alpha_{i, j} \kappa\left(a_{i} \otimes u_{j}\right)=0$ then $\sum \alpha_{i, j} a_{i} \otimes u_{j} \in \operatorname{Im}\left(q_{\Psi}^{3}\right)$. It follows that $\phi$ is injective.

Now, since $\left(q_{\Psi}^{3}\right)^{\top} \circ \kappa=0$, there exists a map $\bar{\kappa}$ such that $m^{\top} \circ \bar{\kappa}=\phi$. This map is bijective since $\phi$ is injective and it is skew-symmetric since $\kappa$ is.

Lemma 6.7 We have the following exact sequences.

$$
\begin{aligned}
& 0 \rightarrow \wedge^{2} U \rightarrow A \otimes E \rightarrow K \rightarrow 0 \\
& 0 \rightarrow M \rightarrow A^{*} \otimes Q^{*} \rightarrow L \rightarrow 0
\end{aligned}
$$

Furthermore, in the notation of Lemmas 6.3 and 6.6, there is a natural isomorphism $\eta: M \simeq N$ which makes the following diagram commutative

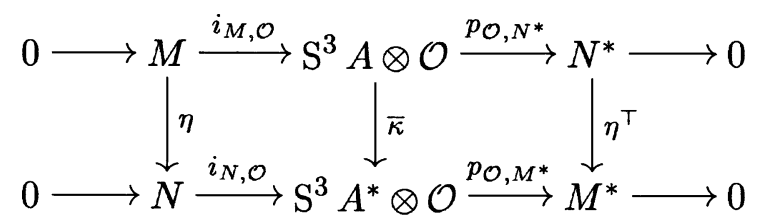

Proof The exact sequence (42) follows immediately by (38) and (27).

It is easy to check that the exact sequences (30), (31), (32), (33) and (34) induce the following exact commutative diagram (omitting surrounding zeroes) 


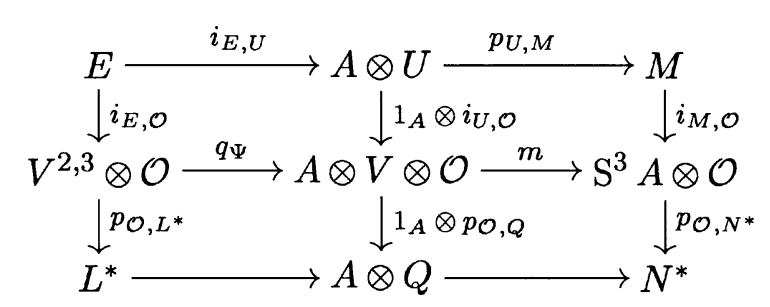

thus the dual of the bottom row provides (43).

Further, since the diagram (41) is commutative, and since the homomorphism $\kappa$ is induced by the map $\psi$ (cfr. Lemma 6.6), we get the following exact commutative diagram (again we omit surrounding zeroes)

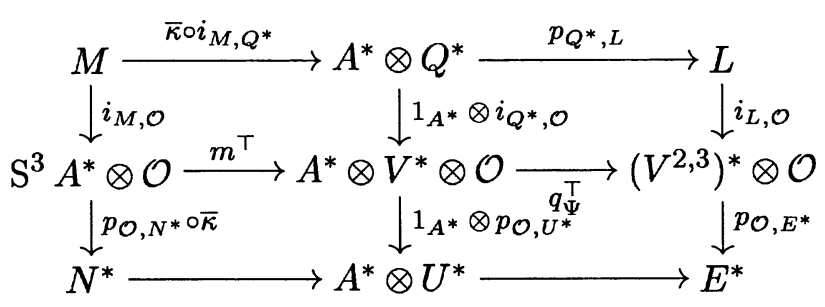

where the central column is the dual of (30), tensorized with $1_{A}$, and the last column is induced by the first two (and in turn it is the same as the dual of (31)).

It is easy to prove that the two maps in the bottom row of (45) (respectively of (46) agree with $i_{L^{*}, Q}$ and $p_{Q, N^{*}}$ (respectively agree with $i_{N^{*}, U^{*}}$ and $\left.p_{U^{*}, E^{*}}\right)$. Since $p_{U^{*}, E^{*}}=i_{E, U}^{\top}$, the bottom row of (46) and the first row of (45) give the isomorphism $\eta$. It is clear also that $\eta$ is induced by $\bar{\kappa}$, so that (44) is commutative.

Tracing back the above proof, it is straightforward to prove the following corollary.

Corollary 6.8 There are the following natural isomorphisms

$$
\begin{aligned}
& \operatorname{Hom}(E, K) \simeq A \\
& \operatorname{Hom}\left(E, Q^{*}\right) \simeq \operatorname{coker}(\bar{\psi}) \\
& \operatorname{Hom}\left(Q^{*}, L\right) \simeq A^{*} \\
& \operatorname{Hom}\left(M, Q^{*}\right) \simeq A \\
& \operatorname{Hom}(U, L) \simeq \operatorname{coker}(\bar{\psi}) \\
& \operatorname{Hom}(U, M) \simeq A
\end{aligned}
$$

Lemma 6.9 The bundle $E$ is stable aCM with $c_{1}(E)=-1$ and $c_{2}(E)=7$. The bundle $L$ is also stable and $a C M$.

Proof The invariants of $E$ are clear by Lemmas 6.1, 6.3, 6.7. The Cohen-Maculay condition for $E$ follows (42) and Lemma 6.2. The bundle $L$ is also aCM by the dual of (31) since the map $p_{\mathcal{O}, E^{*}}$ is surjective on global sections for any twist.

Stability of $E$ and $L$ is obvious from Lemma 2.4 and (31) since $c_{1}(E)=$ $c_{1}(L)=-1$. 


\section{Resolution of the diagonal}

Define the collection $\left(G_{3}, \ldots, G_{0}\right)=\left(E, U, Q^{*}, \mathcal{O}\right)$.

Lemma 7.1 (Kuznetsov) The collection $\left(G_{3}, \ldots, G_{0}\right)=\left(E, U, Q^{*}, \mathcal{O}\right)$ is strongly exceptional, i.e. $\operatorname{Ext}^{p}\left(G_{j}, G_{i}\right)=0$ if $p>0$ or if $i>j$ and $\operatorname{Hom}\left(G_{i}, G_{i}\right) \simeq \mathbb{C}$.

For the original proof we refer to [19]. However it is easy to reprove Lemma 7.1 using Corollary 6.8 and the exact sequences of Lemmas 6.1, 6.3 and 6.7. The dual collection is defined as $\left(G^{3}, \ldots, G^{0}\right)=(E, K, U, \mathcal{O})$.

Theorem 7.2 The general variety $X$ admits the following resolution of the diagonal

$$
\begin{array}{cccc}
\mathcal{P}_{0} & \mathcal{P}_{1} & \mathcal{P}_{2} & \mathcal{P}_{3} \\
0 \longrightarrow & \longrightarrow \otimes E \stackrel{d_{0}}{\longrightarrow} U \otimes K \stackrel{d_{1}}{\longrightarrow} Q^{*} \otimes U \stackrel{d_{2}}{\longrightarrow} \mathcal{O} \longrightarrow \mathcal{O}_{\Delta}
\end{array}
$$

where the arrows are given by the following natural elements

$$
\begin{aligned}
& \operatorname{Hom}\left(\mathcal{P}_{0}, \mathcal{P}_{1}\right) \simeq A^{*} \otimes A \ni 1_{A} \\
& \operatorname{Hom}\left(\mathcal{P}_{1}, \mathcal{P}_{2}\right) \simeq B \otimes B^{*} \ni 1_{B} \\
& \operatorname{Hom}\left(\mathcal{P}_{2}, \mathcal{P}_{3}\right) \simeq V^{*} \otimes V \ni 1_{V}
\end{aligned}
$$

Proof Let us look at the maps in more detail

$d_{2}:$

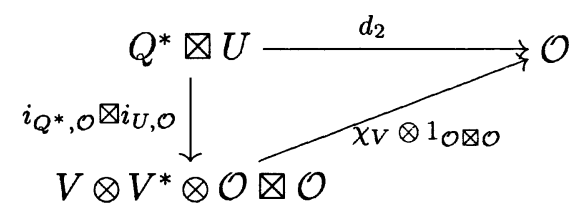

$d_{1}:$
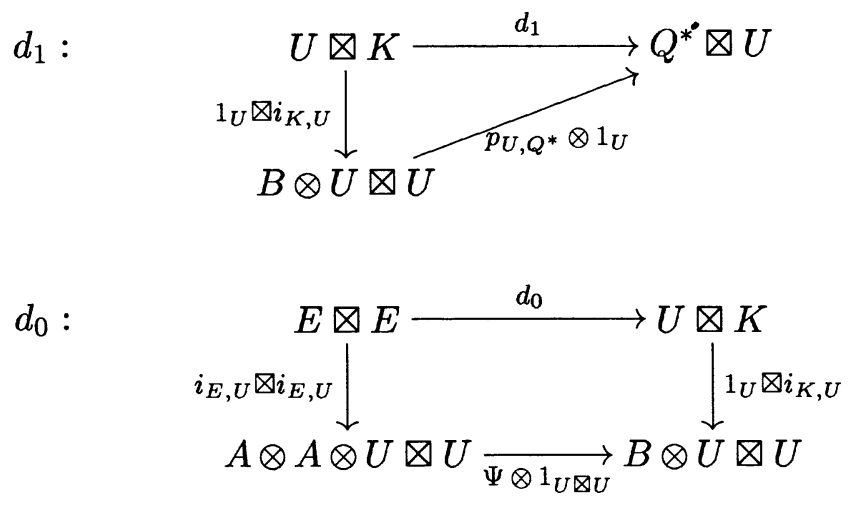

The map $d_{2}$ is the restriction from $\mathrm{G}=\mathbb{G}\left(\mathbb{C}^{3}, V\right)$ of a map $\tilde{d}: U_{\mathrm{G}} \otimes Q_{\mathrm{G}}^{*} \rightarrow$ $\mathcal{O}_{\mathrm{G}}$ and it is a classical fact that $\operatorname{coker}(\tilde{d}) \simeq \mathcal{O}_{\Delta(\mathrm{G})}$. Thus we have $\operatorname{coker}\left(d_{2}\right) \simeq$ $\mathcal{O}_{\Delta}$ and the sequence is exact in $\mathcal{P}_{3}$. 
Let us now look at the composition in $\mathcal{P}_{2}$. It is convenient to prove exactness for the dualized maps which we then write

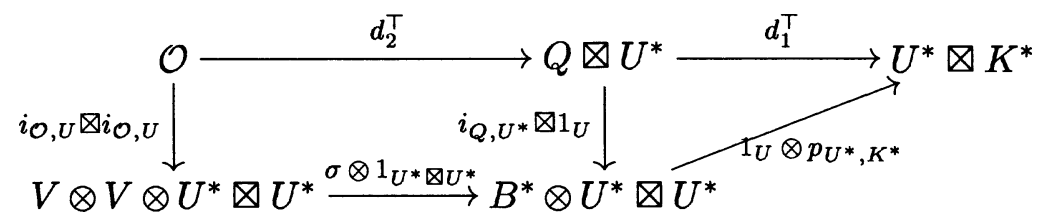

This yields

$$
\operatorname{ker}\left(d_{1}^{\top}\right)=Q \otimes U^{*} \cap U^{*} \otimes Q \subset B^{*} \otimes U^{*} \otimes U^{*}
$$

Then the mixed tensor products can be separated by factoring out the identity over $U^{*}$. If $\tau$ is the involution interchanging factors in $X \times X$, then we have the symmetry $U^{*} \otimes Q=\tau^{*}\left(Q \otimes U^{*}\right)$. So exactness in $\mathcal{P}_{2}$ is proved if we prove surjectivity of the map $\bar{p}$ below

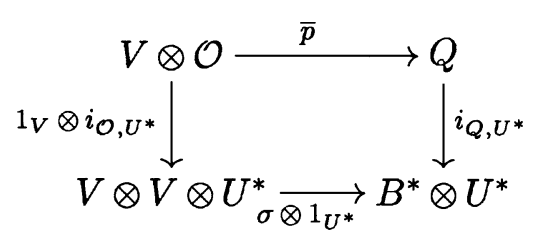

Hence we are done if we prove that the map $\bar{p}$ is the universal quotient (which is clearly surjective), i.e. if we prove that $p_{\mathcal{O}, Q^{*}}$ makes the diagram (54) commutative when replacing $\bar{p}$. And indeed this holds since any morphism $b: U \rightarrow Q^{*}$ comes from a skew-symmetric homomorphism $\bar{b}: V \rightarrow V^{*}$ of the ambient space and we have $\sigma^{\top}(b)(u \wedge v)=\bar{b}(v)(u)$.

Let us turn to $\mathcal{P}_{1}$. Looking at the definition we have

$$
\operatorname{ker}\left(d_{2}\right)=U \otimes K \cap K \otimes U \subset B \otimes U \otimes U
$$

Just as in the case of $\mathcal{P}_{2}$ we are allowed to separate the mixed tensor products by factoring out the identity over $U$ and so reduce to prove surjectivity below

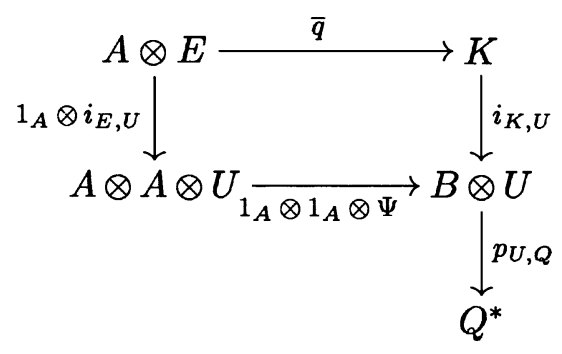

Thus we are done if we prove that the following sequence is exact in $B \otimes U$

$$
0 \rightarrow \wedge^{2} U \rightarrow A \otimes E \stackrel{\Psi \circ i_{E, U}}{\longrightarrow} B \otimes U \stackrel{p_{U, Q^{*}}}{\longrightarrow} Q^{*} \rightarrow 0
$$


But this is proved in Proposition 6.4.

By the classical argument in [3], we get the following corollary.

Corollary 7.3 Any coherent sheaf $\mathcal{F}$ on $X$ is functorially isomorphic to the cohomology a complex $\mathcal{C}_{\mathcal{F}}$ whose terms are given by

$$
\mathcal{C}_{\mathcal{F}}^{k}=\bigoplus_{i-j=k} \mathrm{H}^{i}\left(\mathcal{F} \otimes G^{j}\right) \otimes G_{j}
$$

Alternatively $\mathcal{F}$ is functorially isomorphic to the cohomology a complex $\mathcal{D}_{\mathcal{F}}$ whose terms are given by

$$
\mathcal{D}_{\mathcal{F}}^{k}=\bigoplus_{i-j=k} \mathrm{H}^{i}\left(\mathcal{F} \otimes G_{j}\right) \otimes G^{j}
$$

We have the following standard consequence of Theorem 7.2, namely Castelnuovo-Mumford regularity associated to the collection $\left(G_{3}, \ldots, G_{0}\right)$.

Corollary 7.4 Let $\mathcal{F}$ be a coherent sheaf on $X$ and suppose $\mathrm{H}^{p}\left(G_{p} \otimes \mathcal{F}\right)=0$ for $p>0$. Then $\mathcal{F}$ is globally generated.

Proof Again by a standard argument, one looks at the term $\mathcal{D}_{\mathcal{F}}^{0}$ in the complex $\mathcal{D}_{\mathcal{F}}$, which is isomorphic to $\mathrm{H}^{0}(\mathcal{F}) \otimes \mathcal{O}$ in the hypothesis. On the other hand, in the complex $\mathcal{D}_{\mathcal{F}}$, any differential with source in $\mathrm{H}^{0}(\mathcal{F}) \otimes \mathcal{O}$ vanishes. Therefore the evaluation map $p_{\mathcal{O}, \mathcal{F}}$ is surjective and the statement is proved.

\section{Further remarks}

Remark 8.1 The diagram (55) can be completed to the following exact diagram, where we omit surrounding zeroes for brevity.

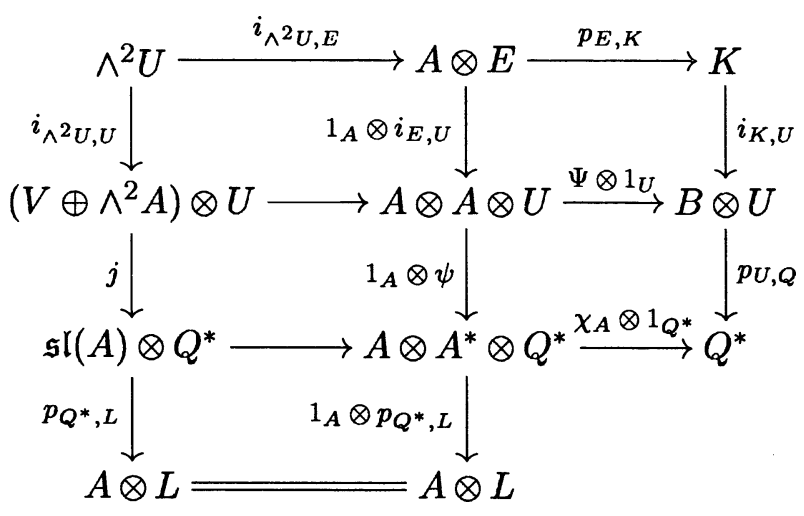

where $j$ is defined by the inclusion $V \oplus \wedge^{2} A \hookrightarrow A \otimes A$ followed by $\psi$, defined by (39) in Remark 6.5. 
Proof Exactness of the horizontal sequences is straightforward. The central column follows from the exact sequences (32) and (43). The right column is (27). The left column is induced by the central and right ones, where the isomorphism $\operatorname{Hom}\left(\wedge^{2} U, U\right) \simeq \operatorname{ker}(A \otimes A \stackrel{\Psi}{\rightarrow} B) \simeq V \oplus \wedge^{2} A$, is clear. Commutativity of all the squares is left to the reader.

\subsection{Helices}

We refer to [1] and [4] for general definitions and properties concerning helices and to [24] for the study of helices on Fano threefolds.

Consider the collection $\left(G_{3}, \ldots, G_{0}\right)$ of Sect. 7 (strongly exceptional by Lemma 7.1) and extend it defining $G_{j+4 k}=G_{j} \otimes \mathcal{O}(-1)$ for any $j=0$, $1,2,3$ and any $k$. We will show in the following remark that $G_{j+1} \simeq$ $\mathrm{L}_{G_{j}} \mathrm{~L}_{G_{j-1}} \mathrm{~L}_{G_{j-2}} G_{j-3}$, for any $j$, according to Definition 2.1. All of the sequences from (56) to (59) below are obtained resolving $G_{i} \otimes \mathcal{O}(1)$ with respect to the basis $\left(G_{3}, \ldots, G_{0}\right)$ according to Corollary 7.3.

Remark 8.2 There are the following exact sequences.

$$
\begin{aligned}
& \mathcal{O}(-1) \stackrel{i_{\mathcal{O}(-1), E}}{\longrightarrow} V^{2,3} \otimes E \stackrel{h_{2}^{0}}{\rightarrow} \operatorname{ker}(\varsigma) \otimes U \stackrel{h_{1}^{0}}{\rightarrow} V \otimes Q^{*} \stackrel{p_{Q^{*}, \mathcal{O}}}{\longrightarrow} \mathcal{O} \\
& Q^{*}(-1) \stackrel{i_{Q^{*}, \mathcal{O}}}{\longrightarrow} V^{*} \otimes \mathcal{O}(-1) \stackrel{h_{2}^{1}}{\rightarrow} A \otimes E \stackrel{h_{1}^{1}}{\rightarrow} B \otimes U \stackrel{p_{U, Q^{*}}}{\longrightarrow} Q^{*} \\
& U(-1) \stackrel{i_{U, Q^{*}}}{\longrightarrow} B^{*} \otimes Q^{*}(-1) \stackrel{h_{2}^{2}}{\rightarrow} \operatorname{ker}(\varsigma)^{*} \otimes \mathcal{O}(-1) \stackrel{h_{1}^{2}}{\rightarrow} A^{*} \otimes E \stackrel{p_{E, U}}{\longrightarrow} U \\
& E \stackrel{i_{E, U}}{\longrightarrow} A \otimes U \stackrel{h_{2}^{3}}{\longrightarrow} A^{*} \otimes Q^{*} \stackrel{h_{1}^{3}}{\rightarrow}\left(V^{2,3}\right)^{*} \otimes \mathcal{O} \stackrel{p_{\mathcal{O}, E^{*}}}{\longrightarrow} E^{*}
\end{aligned}
$$

where the first map in each sequence is injective and the last one is surjective.

In (56) $\varsigma$ is defined in Remark $4.4, h_{1}^{0}$ is the defined by the composition $\operatorname{ker}(\varsigma) \otimes U \hookrightarrow V \otimes B \otimes U$ followed by $1_{V} \otimes p_{U, Q^{*}}$ and $h_{2}^{0}$ is given by

$$
V^{2,3} \otimes E \stackrel{q_{\Psi} \otimes i_{E, U}}{\longrightarrow} V \otimes A \otimes A \otimes U \stackrel{1_{V} \otimes \Psi \otimes 1_{U}}{\longrightarrow} V \otimes B \otimes U
$$

In (57), $h_{1}^{1}$ is the composition

$$
A \otimes E \stackrel{1_{A} \otimes 1_{E, U}}{\longrightarrow} A \otimes A \otimes U \stackrel{\Psi \otimes 1_{U}}{\longrightarrow} B \otimes U
$$

while $h_{2}^{1}$ is identified with the projection $V^{*} \otimes \mathcal{O}(-1) \rightarrow \wedge^{2} U \simeq U^{*}(-1)$.

In (58) we have $\operatorname{ker}\left(p_{E, U}\right) \simeq K^{*}(-1)$ by (42). This gives back the isomorphisms $\operatorname{ker}(\varsigma)^{*} \simeq \operatorname{coker}\left(\varsigma^{\top}\right) \simeq \mathrm{H}^{0}\left(K^{*}\right)$, established in the proof of Lemma 6.1. and the map $h_{1}^{2}$. Then $h_{2}^{2}$ is given by (28).

In (58) we have to glue together the exact sequences (31), (32) and (43). 


\subsection{Quasi-homogeneous case}

In this section we restrict our attention to $U_{22}$, the Mukai-Umemura threefold, i.e. the $S L(2)$-quasi homogeneous case. This is thoroughly studied in $[2,23,21]$. Let us denote $Y_{1}$ the standard representation space of SL(2) and $Y_{n}$ the weight $-n$ representation, so that and $Y_{n}=\mathrm{S}^{n} Y_{1}$.

In terms of plane quartics $U_{22}$ corresponds to a double conic. The action of $\mathrm{SO}(3)$ preserves this conic so we may view $B$ as $Y_{2}$ and the stabilizer in $\mathrm{SO}(3)$ of a polar hexagon is the order 60 icosahedral group, isomorphic to $A_{5}$.

In terms of the net $\Psi$ of dual quadrics, $U_{22}$ corresponds to a net containing a twisted cubic in the dual space, on which SL(2) naturally acts. In this case there are isomorphisms of $\mathrm{SL}(2)$-modules

$$
B \simeq Y_{2} \quad A \simeq Y_{3}
$$

The net of dual quadrics $\Psi$ is itself equivariant. Therefore by the isomorphism $\mathrm{S}^{2} A \simeq Y_{6} \oplus Y_{2}$ we deduce $V \simeq Y_{6}$. Further, the resolution of $R^{\Psi}$ takes the form (3), so one computes $V^{2,3} \simeq Y_{7}$. The instanton $\mathcal{E}_{\Psi}$ of Sect. 3 is endowed with an $\mathrm{SL}(2)$-action in this case and $\mathrm{H}^{1}\left(\Omega_{\mathbb{P}(A)} \otimes \mathcal{E}_{\Psi}\right)$ is isomorphic to $Y_{1} \oplus Y_{5}$.

The threefold $U_{22}$ also corresponds to the (smooth) closure of the SL(2)-orbit of the polynomial $x^{11} y+11 x^{6} y^{6}-x y^{11}$ in $Y_{12}$. This appeared first in [23]. The roots of this polynomial can be drawn in the Riemann sphere to form the vertices of a regular icosahedron. For a quick sketch of how this relates to the other Fano threefolds with $b_{3}=0$ see also [11].

Proposition 8.3 The variety $U_{22}$ admits the resolution of the diagonal (53), where all maps are $\mathrm{SL}(2)$-equivariant.

Proof The maps we have defined over the product $X \times X$ in Theorem 7.2 are equivariant under the $S L(2)$-action. Since $d_{i}$ represents the identity in $\operatorname{Hom}_{X \times X}\left(\mathcal{P}_{i}, \mathcal{P}_{i+1}\right) \simeq Y_{w(i)} \otimes Y_{w(i)}$, where $w(0)=3, w(1)=2, w(2)=6$, it lies in the unique 1-dimensional SL(2)-invariant subspace of $\operatorname{Hom}_{X \times X}\left(\mathcal{P}_{i}, \mathcal{P}_{i+1}\right)$.

Computing the weights of $\operatorname{Hom}_{X \times X}\left(\mathcal{P}_{i}, \mathcal{P}_{i+2}\right)$, one sees that there are no $\mathrm{SL}(2)$-invariant subspaces. Thus the composition $d_{i} \circ d_{i-1}$ is zero for all $i$.

The sequence (8) in this case can be read in terms of SL(2)-modules and it boils down to

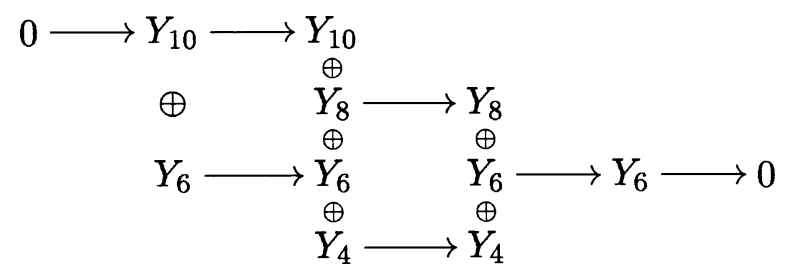

This sequence is clearly exact. However the proof of exactness in (55) is forced since the induced map $A \otimes E \rightarrow K$ is SL(2)-invariant, hence it coincides (up to a scalar) with the projection from $A \otimes E$ onto the cokernel of $\wedge^{2} U \rightarrow A \otimes E$ and as such it surjective. 
Since all the maps defined in Theorem 7.2 are functorial, they lift to the moduli space of $V_{22}$ threefolds. So, once we prove that the sequence of morphisms (53) is a complex, by semicontinuity we can deduce general exactness from exactness over a point of the moduli space. By the above proposition this point can be taken to be $\left[U_{22}\right]$.

Acknowledgements I would like to express my gratitude to Enrique Arrondo for many useful discussions and Frank-Olaf Schreyer and Giorgio Ottaviani for interesting comments. Also, I would like to thank the Department of Mathematics of Universidad Complutense de Madrid for the hospitality. The author was partially supported by Italian MIUR funds and the EAGER contract HPRN-CT-2000-00099.

\section{References}

1. King, A.D., Kobak, P., Maciocia, A. (eds.): Helices and vector bundles, vol. 148 of London Mathematical Society Lecture Note Series. Cambridge University Press, Cambridge (1990) Seminaire Rudakov, Translated from the Russian

2. Aluffi, P., Faber, C.: Linear orbits of $d$-tuples of points in $\mathbf{P}^{1}$. J. Reine Angew. Math. 445, 205-220 (1993)

3. Beilinson, A.A.: Coherent sheaves on $\mathbf{P}^{n}$ and problems in linear algebra. Funktsional. Anal. i Prilozhen. 12(3), 68-69 (1978). English Funct. Anal. Appl. 12, 214-216 (1979) (English)

4. Bondal, A.I.: Helices, representations of quivers and Koszul algebras. In: Helices and vector bundles, vol. 148 of London Math. Soc. Lecture Note Ser., pp. 75-95. Cambridge Univ. Press, Cambridge (1990)

5. Bott, R.: Homogeneous vector bundles. Ann. of Math. 66(2), 203-248 (1957)

6. Canonaco, A.: A Beilinson-type theorem for coherent sheaves on weighted projective spaces. J. Algebra 225(1), 28-46 (2000)

7. Drezet, J.-M.: Fibrés exceptionnels et suite spectrale de Beilinson généralisée sur $\mathbf{P}_{2}(\mathbf{C})$. Math. Ann. 275(1), 25-48 (1986)

8. Drezet, J.-M.: Cohomologie des variétés de modules de hauteur nulle. Math. Ann. 281(1), 43-85 (1988)

9. Eisenbud, D.: Commutative algebra, vol. 150 of Graduate Texts in Mathematics. SpringerVerlag, New York (1995) With a view toward algebraic geometry

10. Ellingsrud, G., Piene, R., Strømme, S.A.: On the variety of nets of quadrics defining twisted cubics. In: Space curves (Rocca di Papa, 1985), vol. 1266 of Lecture Notes in Math., pp. 8496. Springer, Berlin (1987)

11. Faenzi, D.: Bundles over the Fano threefold $V_{5}$. Communications in Algebra 33, 30613080, 2005 (2003)

12. Gorodentsev, A.L., Rudakov, A.N.: Exceptional vector bundles on projective spaces. Duke Math. J. 54(1), 115-130 (1987)

13. Gruson, L., Skiti, M.: 3-instantons et réseaux de quadriques. Math. Ann. 298(2), 253-273 (1994)

14. Huybrechts, D., Lehn, M.: The geometry of moduli spaces of sheaves. Aspects of Mathematics, E31. Friedr. Vieweg \& Sohn, Braunschweig (1997)

15. Iskovskih, V.A.: Fano threefolds. I. Izv. Akad. Nauk SSSR Ser. Mat. 41(3), 516-562, 717 (1977)

16. Iskovskih, V.A.: Fano threefolds. II. Izv. Akad. Nauk SSSR Ser. Mat. 42(3), 506-549 (1978)

17. Iskovskikh, V.A., Prokhorov, Y.G.: Fano varieties. In: Algebraic geometry, V, vol. 47 of Encyclopaedia Math. Sci., pp. 1-247. Springer, Berlin (1999)

18. Kapranov, M.M.: On the derived categories of coherent sheaves on some homogeneous spaces. Invent. Math. 92(3), 479-508 (1988)

19. Kuznetsov, A.G.: An exceptional set of vector bundles on the varieties $V_{22}$. Vestnik Moskov. Univ. Ser. I Mat. Mekh. 92(3), 41-44 (1996)

20. Kuznetsov, A.G.: Fano threefolds $V_{22}$. preprint MPI 1997-24 (1997) 
21. Mukai, S.: Fano 3-folds. In: Complex projective geometry (Trieste, 1989/Bergen, 1989), vol. 179 of London Math. Soc. Lecture Note Ser., pp. 255-263. Cambridge Univ. Press, Cambridge (1992)

22. Mukai, S.: Plane quartics and fano threefolds of genus twelve. RIMS preprint 1422 (2003)

23. Mukai, S., Umemura, H.: Minimal rational threefolds. In: Algebraic geometry (Tokyo/Kyoto, 1982), vol. 1016 of Lecture Notes in Math., pp. 490-518. Springer, Berlin (1983)

24. Nogin, D.Y.: Helices on some Fano threefolds: constructivity of semiorthogonal bases of $K_{0}$. Ann. Sci. École Norm. Sup. (4) 27(2), 129-172 (1994)

25. Okonek, C., Schneider, M., Spindler, H.: Vector bundles on complex projective spaces, vol. 3 of Progress in Mathematics. Birkhäuser Boston, Mass. (1980)

26. Orlov, D.O.: Exceptional set of vector bundles on the variety $V_{5}$. Vestnik Moskov. Univ. Ser. I Mat. Mekh. (5), 69-71 (1991)

27. Schreyer, F.-O.: Geometry and algebra of prime Fano 3-folds of genus 12. Compositio Math. 127(3), 297-319 (2001) 